\title{
Leapfrog Constructions: From Continuant Polynomials to Permanents of Matrices
}

\author{
Alberto Facchini* \\ Dipartimento di Matematica \\ Università di Padova \\ Padova, Italy \\ facchini@math.unipd.it
}

\author{
André Leroy \\ Faculté Jean Perrin \\ Université d'Artois \\ Lens, France \\ andre.leroy@univ-artois.fr
}

Submitted: Aug 26, 2014; Accepted: Jan 20, 2015; Published: Feb 16, 2015

Mathematics Subject Classifications: Primary 05B45, 11B39; Secondary 15A15

\begin{abstract}
We study noncommutative continuant polynomials via a new leapfrog construction. This needs the introduction of new indeterminates and leads to generalizations of Fibonacci polynomials, Lucas polynomials and other families of polynomials. We relate these polynomials to various topics such as quiver algebras and tilings. Finally, we use permanents to give a broad perspective on the subject.
\end{abstract}

\section{Introduction}

The continuants (or continuant polynomials) $p_{n}$ were introduced in the noncommutative setting in [6] by P.M. Cohn, who used them to describe some groups of invertible matrices and, under suitable hypotheses, to analyze comaximal relations in a ring. See [5] for more details. Continuants also appear in connection with the weak algorithm [5] and more recently they have been used to characterize Euclidean pairs and quasi Euclidean rings [1]. Cohn also calls the construction of continuants leapfrog construction.

In this paper, we prefer to present Cohn's construction with a different notation, which we think is more convenient, and which highlights the leapfrog structure of the construction of the polynomials $p_{n}$ (Section 2). We reinterpret the continuants $p_{n}$ in term of suitable quiver algebras with two vertices $A$ and $B$, in which the paths leap alternatively from $A$ to $B$. Cohn's polynomials $p_{n}$ are related to the elementary group $E_{2}(R)$ (equation (8)). Here we find the leapfrog structure again, because the $n$-th powers of a suitable

*Partially supported by Università di Padova (Progetto ex 60\% "Anelli e categorie di moduli") and Fondazione Cassa di Risparmio di Padova e Rovigo (Progetto di Eccellenza "Algebraic structures and their applications".) 
coset $E_{2}(R) P(0)$ of an element $P(0)$ of $G L_{n}(R)$ modulo $E_{2}(R)$ jump alternatively from $E_{2}(R)$ when $n$ is even to $E_{2}(R) P(0)$ when $n$ is odd.

In a completely different setting, other polynomials $h_{n}$ were introduced in [2], to compute the inverse of an isomorphism $f$ in a factor category $\mathcal{A} / \mathcal{I}_{1} \cap \cdots \cap \mathcal{I}_{n}$ from the inverses of the images of the isomorphism $f$ in factor categories $\mathcal{A} / \mathcal{I}_{t}$ of $\mathcal{A}$ modulo ideals $\mathcal{I}_{t}$ of $\mathcal{A}$. Since there is a surprising similarity between the structure of Cohn's polynomials $p_{n}$ and that of the polynomials $h_{n}$, we have studied whether both families of polynomials were specializations of a more general class of polynomials. In this investigation, we have met with other classes of noncommutative polynomials (the generalized Fibonacci polynomials $f_{n}$, the generalized Lucas polynomials $\ell_{n}$ and other "circular" polynomials $c_{n}$ ), which have evident combinatorial interpretations: they parametrize tilings and circular tilings of stripes of squares with dominoes (Section 7). Commutative generalized Fibonacci polynomials appear naturally in Combinatorics [3], related to the Fibonacci sequence, and in Complex Analysis [7], related to generalized continuous fractions. Our noncommutative generalized Fibonacci polynomials are the noncommutative analogue of the generalized Fibonacci polynomials studied in [3].

We have thus found a very natural "hierarchy" of polynomials (see the diagram in Remark 16(4)). Our polynomials turn out to be noncommutative permanents of suitable matrices in noncommutative indeterminates (Theorem 17 and Corollary 18).

The rings and algebras we work with are associative and have an identity element.

\section{Two sets of indeterminates in Cohn's continuants $\boldsymbol{p}_{n}$}

We begin this Section by recalling the definition of the continuants [6], which are noncommutative polynomials with coefficients in the ring $\mathbb{Z}$ of integers. Let $t_{1}, t_{2}, t_{3}, \ldots$ be infinitely many noncommutative indeterminates over the ring $\mathbb{Z}$. There is a strictly ascending chain

$$
\mathbb{Z}\left\langle t_{1}\right\rangle \subset \mathbb{Z}\left\langle t_{1}, t_{2}\right\rangle \subset \mathbb{Z}\left\langle t_{1}, t_{2}, t_{3}\right\rangle \subset \cdots
$$

of noncommutative integral domains, where $\mathbb{Z}\left\langle t_{1}, \ldots, t_{n}\right\rangle$ denotes the ring of polynomials in the noncommutative indeterminates $t_{1}, \ldots, t_{n}$ with coefficients in $\mathbb{Z}$, and the union of this chain is the ring $\mathbb{Z}\left\langle t_{1}, t_{2}, t_{3}, \ldots\right\rangle$. The continuants $p_{n}=p_{n}\left(t_{1}, \ldots, t_{n}\right)$ are defined by the recursion formulae:

$$
\begin{aligned}
& p_{-1}=0, \quad p_{0}=1, \text { and, for } n \geqslant 1, \\
& p_{n}\left(t_{1}, \ldots, t_{n}\right)=p_{n-1}\left(t_{1}, \ldots, t_{n-1}\right) t_{n}+p_{n-2}\left(t_{1}, \ldots, t_{n-2}\right) .
\end{aligned}
$$

For every $f \in \mathbb{Z}\left\langle t_{1}, t_{2}, t_{3}, \ldots\right\rangle$, we will use P.M. Cohn's convenient notation, introduced in [5, p. 147], denoting the $2 \times 2$-matrix $\left(\begin{array}{ll}f & 1 \\ 1 & 0\end{array}\right)$ by $P(f)$. Then

$$
P\left(t_{1}\right) P\left(t_{2}\right) \ldots P\left(t_{n}\right)=\left(\begin{array}{cc}
p_{n}\left(t_{1}, \ldots, t_{n}\right) & p_{n-1}\left(t_{1}, \ldots, t_{n-1}\right) \\
p_{n-1}\left(t_{2}, \ldots, t_{n}\right) & p_{n-2}\left(t_{2}, \ldots, t_{n-1}\right)
\end{array}\right) .
$$


Since the inverse of the matrix $P(f)$ is given by $P(0) P(-f) P(0)$, we easily get that the inverse of the matrix in (2) is given by

$$
(-1)^{n}\left(\begin{array}{cc}
p_{n-2}\left(t_{n-1}, \ldots, t_{2}\right) & -p_{n-1}\left(t_{n-1}, \ldots, t_{1}\right) \\
-p_{n-1}\left(t_{n}, \ldots, t_{2}\right) & p_{n}\left(t_{n}, \ldots, t_{1}\right)
\end{array}\right)
$$

This leads to the following well-known relations:

$$
\begin{gathered}
p_{n}\left(t_{1}, \ldots, t_{n}\right) p_{n-1}\left(t_{n-1}, \ldots, t_{2}\right)-p_{n-1}\left(t_{1}, \ldots, t_{n-1}\right) p_{n-1}\left(t_{n}, \ldots, t_{2}\right)=(-1)^{n} \\
p_{n}\left(t_{1}, \ldots, t_{n}\right) p_{n-1}\left(t_{n-1}, \ldots, t_{1}\right)=p_{n-1}\left(t_{1}, \ldots, t_{n-1}\right) p_{n}\left(t_{n}, \ldots, t_{1}\right)
\end{gathered}
$$

This last relation is due to Wedderburn [9].

Using the associativity of the product of matrices in (2), we also get, for any $1 \leqslant k \leqslant n$, the formula:

$$
\begin{aligned}
& p_{n}\left(t_{1}, \ldots, t_{n}\right) \\
& =p_{k}\left(t_{1}, \ldots, t_{k}\right) p_{n-k}\left(t_{n-k+1}, \ldots, t_{n}\right)+p_{k-1}\left(t_{1}, \ldots, t_{k-1}\right) p_{n-k-1}\left(t_{n-k}, \ldots, t_{n}\right) .
\end{aligned}
$$

For $k=1$, this gives back the equation given in [5, (14), page 148]:

$$
p_{n}\left(t_{1}, \ldots, t_{n}\right)=t_{1} p_{n-1}\left(t_{2}, \ldots, t_{n}\right)+p_{n-2}\left(t_{3}, \ldots, t_{n}\right) .
$$

The continuant polynomial $p_{n}\left(t_{1}, \ldots, t_{n}\right)$ can be presented via a leapfrog construction in the following sense. The first term of $p_{n}$ is $t_{1} t_{2} \cdots t_{n}$. The next terms are obtained by erasing two consecutive indeterminates (the frog leaps over them) from $t_{1} t_{2} \cdots t_{n}$ to get the sum: $t_{3} t_{4} \cdots t_{n}+t_{1} t_{4} t_{5} \cdots t_{n}+t_{1} t_{2} t_{5} \cdots t_{n}+\ldots$ As far as the following terms are concerned, we erase 2 pairs of consecutive indeterminates (2 jumps) and get the terms

$$
\sum_{1 \leqslant i_{1}<i_{2}-1 \leqslant n} t_{1} \cdots \widehat{t_{i_{1}}} \widehat{t_{i_{1}+1}} \cdots \widehat{t_{i_{2}}} \widehat{t_{i_{2}+1}} \cdots t_{n}
$$

We then continue adding terms corresponding to 3 leaps, 4 leaps, and so on. Finally, we can write

$$
p_{n}\left(t_{1}, \ldots, t_{n}\right)=\sum_{i_{1}, i_{2}, \ldots, i_{j}} t_{1} \cdots \widehat{t_{i_{1}}} \widehat{t_{i_{1}+1}} \cdots \widehat{\widehat{t_{i_{2}}}} \widehat{t_{i_{2}+1}} \cdots \widehat{t_{i_{j}}} \widehat{t_{i_{j}+1}} \cdots t_{n}
$$

where $1 \leqslant j \leqslant\lfloor n / 2\rfloor$ and $i_{j}+1<i_{j+1}$ for every $j$,

As we have already said in the Introduction, in order to highlight another leapfrog structure of the construction of the polynomials $p_{n}$, we find more convenient to denote the indeterminates $t_{2 n-1}$ with odd index $2 n-1$ by $x_{n}$, the indeterminates $t_{2 n}$ with even index $2 n$ by $y_{n}$, and, similarly, the continuants $p_{2 n}$ by $G_{n}$ and the continuants $p_{2 n-1}$ by $H_{n}$, so 
that $G_{n}$ is a polynomial in the indeterminates $x_{1}, y_{1}, \ldots, x_{n}, y_{n}$, and $H_{n}$ is a polynomial in the indeterminates $x_{1}, y_{1}, \ldots, x_{n-1}, y_{n-1}, x_{n}$.

In order to convince the reader that our different notation is more expressive than the original one, consider the first continuants $p_{n}$. They are

$$
\begin{aligned}
& p_{0}=1, \quad p_{1}=t_{1}, \quad p_{2}=t_{1} t_{2}+1, \quad p_{3}=t_{1} t_{2} t_{3}+t_{1}+t_{3}, \\
& p_{4}=t_{1} t_{2} t_{3} t_{4}+t_{1} t_{2}+t_{1} t_{4}+t_{3} t_{4}+1, \\
& p_{5}=t_{1} t_{2} t_{3} t_{4} t_{5}+t_{1} t_{2} t_{3}+t_{1} t_{2} t_{5}+t_{1} t_{4} t_{5}+t_{3} t_{4} t_{5}+t_{1}+t_{3}+t_{5}, \\
& \begin{array}{c}
p_{6}=t_{1} t_{2} t_{3} t_{4} t_{5} t_{6}+t_{1} t_{2} t_{3} t_{4}+t_{1} t_{2} t_{3} t_{6}+t_{1} t_{2} t_{5} t_{6}+t_{1} t_{4} t_{5} t_{6}+t_{3} t_{4} t_{5} t_{6} \\
\quad+t_{1} t_{2}+t_{1} t_{4}+t_{1} t_{6}+t_{3} t_{4}+t_{3} t_{6}+t_{5} t_{6}+1, \ldots
\end{array}
\end{aligned}
$$

It is rather difficult to recognize the symmetry and the pattern of the polynomials $p_{n}$. But notice that the monomials in $p_{i}$ are of even degree if $i$ is even and of odd degree if $i$ is odd. The same polynomials with the new notation become

$$
\begin{aligned}
& G_{0}=1, \quad G_{1}=x_{1} y_{1}+1, \quad G_{2}=x_{1} y_{1} x_{2} y_{2}+x_{1} y_{1}+x_{1} y_{2}+x_{2} y_{2}+1 \\
& G_{3}=x_{1} y_{1} x_{2} y_{2} x_{3} y_{3}+x_{1} y_{1} x_{2} y_{2}+x_{1} y_{1} x_{2} y_{3}+x_{1} y_{1} x_{3} y_{3} \\
& \quad+x_{1} y_{2} x_{3} y_{3}+x_{2} y_{2} x_{3} y_{3}+x_{1} y_{1}+x_{1} y_{2}+x_{1} y_{3}+x_{2} y_{2}+x_{2} y_{3}+x_{3} y_{3}+1
\end{aligned}
$$

and

$$
\begin{aligned}
& H_{0}=0, \quad H_{1}=x_{1}, \quad H_{2}=x_{1} y_{1} x_{2}+x_{1}+x_{2}, \\
& H_{3}=x_{1} y_{1} x_{2} y_{2} x_{3}+x_{1} y_{1} x_{2}+x_{1} y_{1} x_{3}+x_{1} y_{2} x_{3}+x_{2} y_{2} x_{3}+x_{1}+x_{2}+x_{3} .
\end{aligned}
$$

The pattern with the notation $x_{i}, y_{i}, G_{i}$ and $H_{i}$ is much clearer.

Moreover, the ring $\mathbb{Z}\left\langle t_{1}, t_{2}, t_{3}, \ldots\right\rangle$ is 2 -graded, that is, graded over the group $\mathbb{Z} / 2 \mathbb{Z}$, because every polynomial is the sum of a sum of monomials of even degree and a sum of monomials of odd degree. The polynomials $G_{n}$ turn out to be homogeneous of degree $\overline{0} \in \mathbb{Z} / 2 \mathbb{Z}$, and the polynomials $H_{n}$ turn out to be homogeneous of degree $\overline{1} \in \mathbb{Z} / 2 \mathbb{Z}$. The reason of this lies in the defining recursion relation $p_{n}=p_{n-1} t_{n}+p_{n-2}$. Here the indices $n$ and $n-2$ in $p_{n}$ and $p_{n-2}$ have the same parity. The index $n-1$ in $p_{n-1}$ has different parity, but the degree of $p_{n-1} t_{n}$ has the same parity as $p_{n}$ and $p_{n-2}$.

From

$$
P\left(x_{i}\right) P\left(y_{i}\right)=\left(\begin{array}{cc}
x_{i} y_{i}+1 & x_{i} \\
y_{i} & 1
\end{array}\right)
$$

it follows that

$$
\begin{aligned}
\left(\begin{array}{cc}
x_{1} y_{1}+1 & x_{1} \\
y_{1} & 1
\end{array}\right) \ldots\left(\begin{array}{cc}
x_{n} y_{n}+1 & x_{n} \\
y_{n} & 1
\end{array}\right)= \\
\\
\left(\begin{array}{cc}
G_{n}\left(x_{1}, y_{1}, \ldots, x_{n}, y_{n}\right) & H_{n}\left(x_{1}, y_{1} \ldots, y_{n-1}, x_{n}\right) \\
H_{n}\left(y_{1}, x_{2}, \ldots, x_{n}, y_{n}\right) & G_{n-1}\left(y_{1}, x_{2}, \ldots, y_{n-1}, x_{n}\right)
\end{array}\right) .
\end{aligned}
$$

Since, for any $f \in \mathbb{Z}\left\langle x_{1}, y_{1}, \ldots\right\rangle$, we have

$$
\left(\begin{array}{ll}
f & 1 \\
1 & 0
\end{array}\right)^{-1}=P(0) P(-f) P(0)=\left(\begin{array}{cc}
0 & 1 \\
1 & -f
\end{array}\right)
$$


we easily conclude that the matrix on the right in equation (8) is invertible. Using the remarks about the parity of the degree of the homogeneous polynomials $G_{n}$ and $H_{n}$, we get that the inverse of the matrix on the right in equation (8) is

$$
\left(\begin{array}{cc}
G_{n-1}\left(x_{n}, y_{n-1}, \ldots, y_{1}\right) & -H_{n}\left(x_{n}, \ldots, y_{1}, x_{1}\right) \\
-H_{n}\left(y_{n}, x_{n}, \ldots, y_{1}\right) & G_{n}\left(y_{n}, x_{n}, \ldots, y_{1}, x_{1}\right)
\end{array}\right)
$$

From this inverse, the interested reader can easily obtain the equations analogous to the ones in (4) and (5).

The recursion formulae for $p_{n}$ translate into the following ones between the $G_{n}, H_{n}, x_{n}$ and $y_{n}$ :

$$
G_{n+1}=H_{n+1} y_{n+1}+G_{n} \text { and } H_{n+1}=G_{n} x_{n+1}+H_{n} .
$$

Using the associativity of the product of matrices, we easily obtain the following formulas, for every $1 \leqslant k \leqslant n$ :

$$
\begin{aligned}
G_{n}\left(x_{1}, \ldots, y_{n}\right)=G_{k}\left(x_{1}, \ldots\right. & \left., x_{k-1}, y_{k}\right) G_{n-k}\left(x_{k+1}, \ldots, y_{n}\right) \\
& +H_{k}\left(x_{1}, \ldots, y_{k-1}, x_{k}\right) H_{n-k}\left(y_{k+1}, x_{k+2} \ldots, y_{n}\right)
\end{aligned}
$$

and

$$
\begin{aligned}
H_{n}\left(x_{1}, y_{1}, \ldots, x_{n}\right)=G_{k}\left(x_{1}, \ldots, y_{k}\right) H_{n-k}\left(x_{k}, y-k, \ldots, x_{n}\right) \\
+H_{k}\left(x_{1}, y_{1}, \ldots, x_{k}\right) G_{n-k-1}\left(y_{n-k}, \ldots, y_{n-1}, x_{n}\right) .
\end{aligned}
$$

All the polynomials $p_{n}, H_{n}$ and $G_{n}$ are sums of monomials with all the coefficients equal to 1 . From the defining relations (10), it is easily seen that each $G_{n}$ is a sum of monomials of all possible even degrees $\leqslant 2 n$ and each $H_{n}$ is a sum of monomials of all possible odd degrees $\leqslant 2 n-1$. Also, the number $m_{n}$ of monomials in $p_{n}$, which is clearly equal to $p_{n}(1,1, \ldots, 1)$, satisfies the relations $m_{0}=1, m_{1}=1, m_{2}=2, m_{n}=m_{n-1}+m_{n-2}$, hence $m_{n}=F_{n+1}$, the $(n+1)$-th Fibonacci number, defined by $F_{0}=0, F_{1}=1$ and $F_{n}=F_{n-1}+F_{n-2}$ for $n \geqslant 2$.

Now consider the following directed graph (quiver) $\Gamma_{n}$ with two vertices $A$ and $B$ :

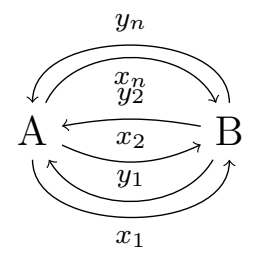

For each $i=1,2, \ldots, n$, the directed graph $\Gamma_{n}$ has one arrow $x_{i}$ from $A$ to $B$, and one arrow $y_{i}$ from $B$ to $A$. Thus $\Gamma_{n}$ has $2 n$ arrows.

Let $k$ be a field. Consider the quiver algebra $k \Gamma_{n}$ and the ideal $I$ of $k \Gamma_{n}$ generated by all paths $x_{i} y_{j}: A \stackrel{x_{i}}{\longrightarrow} B \stackrel{y_{j}}{\longrightarrow} A$ with $i>j$ and all paths $y_{i} x_{j}: B \stackrel{y_{i}}{\longrightarrow} A \stackrel{x_{j}}{\longrightarrow} B$ with 
$i \geqslant j$. The quotient algebra $k \Gamma_{n} / I$ is a finite dimensional $k$-algebra, because the longest possible path not in $I$ is the path $x_{1} y_{1} x_{2} y_{2} \ldots x_{n} y_{n}$ of length $2 n$. In particular, $R:=k \Gamma_{n} / I$ is an artinian ring, so that the Jacobson radical $J(R)$ is a nilpotent ideal that contains all nilpotent elements of $R$. Moreover, $R / J(R) \cong k \times k$. The algebra $R=R_{0} \oplus R_{1}$ is 2-graded, where $R_{0}$ corresponds to the paths of even length and $R_{1}$ to the paths of odd length. In particular, the images of the polynomials $G_{n}$ in $R$ are in $R_{0}$ and the images of the polynomials $H_{n}$ are in $R_{1}$. Notice that $G_{n}$ is a linear combination of paths from $A$ to $A$ (i. e., cycles), and $H_{n}$ is a linear combination of paths from $A$ to $B$. The elements $x_{i} y_{j}$ with $i \geqslant j$ are nilpotent of index 2 , hence they belong to $J(R)$, so $\sum_{1 \leqslant i \leqslant j \leqslant n} x_{i} y_{j}$ is nilpotent, and $1-\sum_{1 \leqslant i \leqslant j \leqslant n} x_{i} y_{j}$ is invertible in $R$. Here, and in the next Proposition, the same symbol denotes both an element of $k \Gamma_{n}$ and its image in $R$.

Theorem 1. In the ring $R=k \Gamma_{n} / I$, we have that

$$
H_{n}=\left(1-\sum_{1 \leqslant i \leqslant j \leqslant n} x_{i} y_{j}\right)^{-1}\left(\sum_{i=1} x_{i}\right) \quad \text { and } \quad G_{n}=\left(1-\sum_{1 \leqslant i \leqslant j \leqslant n} x_{i} y_{j}\right)^{-1}
$$

for every $n \geqslant 0$.

Proof. We claim that $H_{n}=G_{n}\left(\sum_{i=1}^{n} x_{i}\right)$ and $G_{n}\left(1-\sum_{1 \leqslant i \leqslant j \leqslant n} x_{i} y_{j}\right)=1$ in $R$ for every $n \geqslant 1$. The proof of the claim is by induction on $n$. For $n=1$, we have $G_{1} x_{1}=$ $\left(x_{1} y_{1}+1\right) x_{1}=x_{1}=H_{1}$ and $G_{1}\left(1-x_{1} y_{1}\right)=\left(1+x_{1} y_{1}\right)\left(1-x_{1} y_{1}\right)=1$, because $y_{1} x_{1} \in I$. Now assume that the claim is true for $n$ in $R:=k \Gamma_{n} / I$. We will show that the claim is also true for $n+1$ in $R^{\prime}:=k \Gamma_{n+1} / I^{\prime}$. Using the relations (10), we have

$$
\begin{aligned}
H_{n+1}-G_{n+1}\left(\sum_{i=1}^{n+1} x_{i}\right) & =\left(G_{n} x_{n+1}+H_{n}\right)-\left(H_{n+1} y_{n+1}+G_{n}\right)\left(\sum_{i=1}^{n+1} x_{i}\right) \\
& =G_{n} x_{n+1}+H_{n}-G_{n}\left(\sum_{i=1}^{n+1} x_{i}\right) \\
& =H_{n}-G_{n}\left(\sum_{i=1}^{n} x_{i}\right)=0 .
\end{aligned}
$$

This proves the first formula in the claim for $n+1$, i. e., that

$$
H_{n+1}=G_{n+1}\left(\sum_{i=1}^{n+1} x_{i}\right)
$$

in $R^{\prime}$. But $\left(G_{n+1}-G_{n}\right)\left(\sum_{i=1}^{n+1} x_{i}\right)=H_{n+1} y_{n+1}\left(\sum_{i=1}^{n+1} x_{i}\right)=0$, so that we also have $H_{n+1}=G_{n}\left(\sum_{i=1}^{n+1} x_{i}\right)$ in $R^{\prime}$. Finally, in $R^{\prime}:=k \Gamma_{n+1} / I^{\prime}$, we have that

$$
\begin{aligned}
G_{n+1}(1 & \left.-\sum_{1 \leqslant i \leqslant j \leqslant n+1} x_{i} y_{j}\right) \\
& =\left(H_{n+1} y_{n+1}+G_{n}\right)\left(1-\sum_{1 \leqslant i \leqslant j \leqslant n} x_{i} y_{j}-\sum_{i=1}^{n+1} x_{i} y_{n+1}\right) \\
& =H_{n+1} y_{n+1}+G_{n}\left(1-\sum_{1 \leqslant i \leqslant j \leqslant n} x_{i} y_{j}\right)-G_{n}\left(\sum_{i=1}^{n+1} x_{i} y_{n+1}\right) \\
& =1+\left(H_{n+1}-G_{n}\left(\sum_{i=1}^{n+1} x_{i}\right)\right) y_{n+1}=1 .
\end{aligned}
$$


This concludes the proof of the claim. The statement of the Proposition for $n \geqslant 1$ follows immediately from the claim. The case $n=0$ is trivial.

This theorem again shows that the structure of the $H_{n}$ 's is different from the structure of the $G_{n}$ 's, so that the choice of using different notations for the two families of polynomials is appropriate.

Notice that, in our graph $\Gamma_{n}$ with two vertices, the paths leap alternatively from $A$ to $B$.

\section{Continuants and groups of matrices}

For any fixed ring $R$, let $G L_{2}(R)$ denote the group of all invertible $2 \times 2$-matrices with entries in $R$, that is, $G L_{2}(R)=U\left(M_{2}(R)\right)$, the group of units of the ring $M_{2}(R)$ of all $2 \times 2$-matrices with entries in $R$. Let $E_{2}(R)$ be the elementary group, that is, the subgroup of $G L_{2}(R)$ generated by all triangular matrices $\left(\begin{array}{ll}1 & x \\ 0 & 1\end{array}\right)$ and all triangular matrices $\left(\begin{array}{ll}1 & 0 \\ y & 1\end{array}\right)$, where $x$ and $y$ range in $R$. Notice that the triangular matrices $\left(\begin{array}{ll}1 & x \\ 0 & 1\end{array}\right)$ and $\left(\begin{array}{ll}1 & 0 \\ y & 1\end{array}\right)$ in $G L_{2}(R)$ generate $E_{2}(R)$ as a semigroup, because the inverse of the triangular matrix $\left(\begin{array}{ll}1 & x \\ 0 & 1\end{array}\right)$ is $\left(\begin{array}{cc}1 & -x \\ 0 & 1\end{array}\right)$, and similarly for $\left(\begin{array}{ll}1 & 0 \\ y & 1\end{array}\right)$. Since the matrices of the type $\left(\begin{array}{ll}1 & x \\ 0 & 1\end{array}\right)$ form an abelian group isomorphic to the additive group of $R$, and similarly for the matrices $\left(\begin{array}{ll}1 & 0 \\ y & 1\end{array}\right)$, an arbitrary element of $E_{2}(R)$ is a product of finitely many elements of the form

$$
\left(\begin{array}{ll}
1 & x \\
0 & 1
\end{array}\right)\left(\begin{array}{ll}
1 & 0 \\
y & 1
\end{array}\right)=\left(\begin{array}{cc}
x y+1 & x \\
y & 1
\end{array}\right)
$$

These are exactly the factors on the left in the equation (8). Thus an arbitrary element of $E_{2}(R)$ is a matrix of the form

$$
\left(\begin{array}{cc}
G_{n}\left(x_{1}, y_{1}, \ldots, x_{n}, y_{n}\right) & H_{n}\left(x_{1}, y_{1} \ldots, y_{n-1}, x_{n}\right) \\
H_{n}\left(y_{1}, x_{2}, \ldots, x_{n}, y_{n}\right) & G_{n-1}\left(y_{1}, x_{2}, \ldots, y_{n-1}, x_{n}\right)
\end{array}\right)
$$

with $x_{1}, y_{1}, \ldots, x_{n}, y_{n} \in R$.

Let $G$ be the subsemigroup of the multiplicative semigroup $M_{2}(R)$ generated by all matrices of type

$$
P(x):=\left(\begin{array}{cc}
x & 1 \\
1 & 0
\end{array}\right),
$$

where $x$ ranges in $R$. As Cohn proved in [6], the semigroup $G$, set of all products $P\left(x_{1}\right) \cdots P\left(x_{n}\right)$ with $n \geqslant 1$ and $x_{1}, \ldots, x_{n} \in R$, is a group, because $P(0)^{2}$ is the identity of $G L_{2}(R)$ and $P(x)^{-1}=P(0) P(-x) P(0)$. 
Theorem 2. For any ring $R$, exactly one of the following two conditions holds:

(a) Either $G=E_{2}(R)$, or

(b) The group $G$ is the semidirect product $E_{2}(R) \rtimes C$ of the group $E_{2}(R)$ and the cyclic group $C$ of order 2 generated by the involution $P(0)=\left(\begin{array}{ll}0 & 1 \\ 1 & 0\end{array}\right)$.

The action of $P(0)$ on $E_{2}(R)$ is given by

$$
\left(\begin{array}{ll}
1 & x \\
0 & 1
\end{array}\right) \mapsto P(0)\left(\begin{array}{ll}
1 & x \\
0 & 1
\end{array}\right) P(0)=\left(\begin{array}{ll}
1 & 0 \\
x & 1
\end{array}\right)
$$

and

$$
\left(\begin{array}{ll}
1 & 0 \\
y & 1
\end{array}\right) \mapsto P(0)\left(\begin{array}{ll}
1 & 0 \\
y & 1
\end{array}\right) P(0)=\left(\begin{array}{ll}
1 & y \\
0 & 1
\end{array}\right)
$$

Proof. Suppose $G \neq E_{2}(R)$. Then $\left(\begin{array}{ll}1 & x \\ 0 & 1\end{array}\right)=P(x) P(0)$ and $\left(\begin{array}{ll}1 & 0 \\ y & 1\end{array}\right)=P(0) P(y)$, so that $E_{2}(R)$ is contained in $G$. It is easily verified that the action of $P(0)$ on $E_{2}(R)$, given by conjugation by the involution $P(0)$, is as in the last part of the statement of the Theorem. Since every generator $P(x)$ of $G$ can be written as $P(x)=\left(\begin{array}{ll}1 & x \\ 0 & 1\end{array}\right) P(0)$, where $\left(\begin{array}{ll}1 & x \\ 0 & 1\end{array}\right) \in E_{2}(R)$, it follows that $E_{2}(R)$ is a normal subgroup of $G$ and $G=$ $E_{2}(R) C$. In order to conclude the proof that $G$ is the semidirect product of $E_{2}(R)$ and $C$, it remains to notice that $P(0) \notin E_{2}(R)$, because $G \neq E_{2}(R)$ and $G=E_{2}(R) C$.

Proposition 3. If $R$ is any ring of characteristic 2 , then $G=E_{2}(R)$.

Proof. If $R$ has characteristic 2 , then, for $x_{1}=0$ and $y_{1}=x_{2}=y_{2}=1$, we have that

$$
\begin{aligned}
P(0)= & \left(\begin{array}{ll}
1 & 0 \\
1 & 1
\end{array}\right)\left(\begin{array}{ll}
0 & 1 \\
1 & 1
\end{array}\right)= \\
& \left(\begin{array}{cc}
x_{1} y_{1}+1 & x_{1} \\
y_{1} & 1
\end{array}\right)\left(\begin{array}{cc}
x_{2} y_{2}+1 & x_{2} \\
y_{2} & 1
\end{array}\right) \in E_{2}(R) .
\end{aligned}
$$

Thus $G=E_{2}(R)$ by Theorem 2 .

In the next proposition, $\operatorname{char}(R)$ denotes the characteristic of the $\operatorname{ring} R$ and $\operatorname{det}(A)$ denotes the determinant of the matrix $A$.

Proposition 4. Let $R$ be a commutative ring. Then:

(a) $E_{2}(R)=\{A \in G \mid \operatorname{det}(A)=1\}$.

(b) $G=E_{2}(R)$ if and only if $\operatorname{char}(R)=2$. 
Proof. As $R$ is a commutative ring, the determinant det: $G \rightarrow U(R)$ is a group morphism, whose kernel contains $E_{2}(R)$. Thus we have three groups $G \supseteq$ ker det $\supseteq E_{2}(R)$. From Theorem 2 , we know that $\left[G: E_{2}(R)\right] \leqslant 2$. Thus we have three possible cases:

First case: $G=$ ker det $\supset E_{2}(R)$. In this case, $P(0) \in G=$ ker det, so that $\operatorname{det}(P(0))=1$, that is, $-1=1$, hence $\operatorname{char}(R)=2$. By Proposition 3, it follows that $G=E_{2}(R)$. This is not true in this first case. Thus this first case can never take place.

Second case: $G \supset$ ker det $=E_{2}(R)$. In this case, $\operatorname{char}(R) \neq 2$ by Proposition 3. Thus (a) and (b) both hold in this second case.

Third case: $G=$ ker det $=E_{2}(R)$. Then (a) is trivially true. Moreover, as in the first case, we have that $P(0) \in$ ker det, so that $\operatorname{det}(P(0))=1$, hence $\operatorname{char}(R)=2$. Thus (b) also holds.

Let us go back to the case of $R$ nonnecessarily commutative. When $G=E_{2}(R) \rtimes C$, we again found a leapfrog structure, because $\left(\begin{array}{cc}t_{i} & 1 \\ 1 & 0\end{array}\right)=\left(\begin{array}{cc}1 & t_{i} \\ 0 & 1\end{array}\right) P(0)$ is an element of the coset $E_{2}(R) P(0)$ of $G$, so that the products on the left of (2) jump alternatively from elements of $E_{2}(R)$ when $n$ is even to elements of the coset $E_{2}(R) P(0)$ when $n$ is odd.

\section{A second sequence of noncommutative polynomials $h_{n}$}

In this Section, we study another sequence of noncommutative polynomials similar to the sequence of continuants $p_{n}$ considered in the previous two sections. In Section 2, we have preferred to present continuants as polynomials in two infinite sets of indeterminates $x_{1}, x_{2}, x_{3}, \ldots$ and $y_{1}, y_{2}, y_{3}, \ldots$ The polynomials $h_{n}$ we will construct now are noncommutative polynomials in the infinite set of indeterminates $x_{1}, x_{2}, x_{3}, \ldots$ plus one more indeterminate $y$. The polynomials $h_{n}$ have been introduced in [2], in the study of semilocal categories, in order to present a sort of Chinese Remainder Theorem that holds in preadditive categories. Let us recall the definition of those polynomials $h_{n}$, adapting the notation to the context of this paper.

In the paper [2], the authors essentially introduce noncommutative polynomials

$$
h_{n}=h_{n}\left(x_{1}, x_{2}, \ldots, x_{n}, y\right)
$$

with coefficients in $\mathbb{Z}, n \geqslant 1$, defined as follows. Let $x_{1}, x_{2}, x_{3}, \ldots, y$ be infinitely many noncommutative indeterminates over the ring $\mathbb{Z}$. Let $\mathbb{Z}\left\langle x_{1}, x_{2}, x_{3}, \ldots, x_{n}, y\right\rangle$ denote the ring of polynomials in the $n+1$ noncommutative indeterminates $x_{1}, x_{2}, x_{3}, \ldots, x_{n}, y$ with coefficients in $\mathbb{Z}$. For each $n \geqslant 1$, there is a unique polynomial $h_{n}=h_{n}\left(x_{1}, x_{2}, \ldots, x_{n}, y\right)$ in $\mathbb{Z}\left\langle x_{1}, x_{2}, \ldots, x_{n}, y\right\rangle$ such that

$$
1+h_{n} y=\left(1+x_{1} y\right)\left(1+x_{2} y\right) \ldots\left(1+x_{n} y\right) .
$$

In fact, such a polynomial $h_{n}$ exists because the product on the right in the equation (13) is of the form " $1+$ monomials that terminate with $y$ ". Moreover, $h_{n}$ is the unique polynomial that satisfies the equation (13), because $\mathbb{Z}\left\langle x_{1}, x_{2}, \ldots, x_{n}, y\right\rangle$ is an integral domain. 
Proposition 5. The polynomials $h_{n}, n \geqslant 1$, have the following properties:

(a) $1+y h_{n}=\left(1+y x_{1}\right)\left(1+y x_{2}\right) \ldots\left(1+y x_{n}\right)$ for every $n \geqslant 1$.

(b) $h_{1}=x_{1}$, and $h_{n}=x_{n}+h_{n-1}\left(1+y x_{n}\right)$ for every $n \geqslant 2$.

(c) For every $n \geqslant 1$,

$$
\begin{aligned}
h_{n}=\sum_{1 \leqslant i \leqslant n} x_{i} & +\sum_{1 \leqslant i_{1}<i_{2} \leqslant n} x_{i_{1}} y x_{i_{2}} \\
& +\sum_{1 \leqslant i_{1}<i_{2}<i_{3} \leqslant n} x_{i_{1}} y x_{i_{2}} y x_{i_{3}}+\cdots+x_{1} y x_{2} y \ldots y x_{n} .
\end{aligned}
$$

Proof. (a) Multiplying the equation (13) by $y$ on the left, we get that

$$
\begin{aligned}
y\left(1+h_{n} y\right)= & y\left(1+x_{1} y\right)\left(1+x_{2} y\right) \ldots\left(1+x_{n} y\right) \\
= & \left(y+y x_{1} y\right)\left(1+x_{2} y\right) \ldots\left(1+x_{n} y\right)=\left(1+y x_{1}\right) y\left(1+x_{2} y\right) \ldots\left(1+x_{n} y\right) \\
= & \left(1+y x_{1}\right)\left(1+y x_{2}\right) y \ldots\left(1+x_{n} y\right) \\
& \quad \vdots \\
= & \left(1+y x_{1}\right)\left(1+y x_{2}\right) \ldots\left(1+y x_{n}\right) y .
\end{aligned}
$$

But

$$
y\left(1+h_{n} y\right)=y+y h_{n} y=\left(1+y h_{n}\right) y,
$$

so that (a) holds because $\mathbb{Z}\left\langle x_{1}, \ldots, x_{n}, y\right\rangle$ is an integral domain.

(b) Induction on $n \geqslant 1$. From the definition of $h_{1}$, we have that $1+h_{1} y=1+x_{1} y$, so $h_{1}=x_{1}$. As far as an arbitrary $n \geqslant 1$ is concerned, we have, from (13), that

$$
\begin{aligned}
1+h_{n+1} y & =\left(1+x_{1} y\right) \ldots\left(1+x_{n+1} y\right)=\left(1+h_{n} y\right)\left(1+x_{n+1} y\right) \\
& =1+h_{n} y+x_{n+1} y+h_{n} y x_{n+1} y
\end{aligned}
$$

from which $h_{n+1}=h_{n}+x_{n+1}+h_{n} y x_{n+1}=x_{n+1}+h_{n}\left(1+y x_{n+1}\right)$.

(c) follows from the equation (13).

Corollary 6. $h_{n}\left(x_{1}, x_{2}, \ldots, x_{n}, y\right)=x_{1}+\left(1+x_{1} y\right) h_{n-1}\left(x_{2}, x_{3} \ldots, x_{n}, y\right)$

Proof. From Proposition 5(a), applied to $n+1$ and $n$, we have that

$$
\begin{aligned}
1+y h_{n+1}= & \left(1+y x_{1}\right)\left(1+y x_{2}\right) \ldots\left(1+y x_{n+1}\right) \\
& =\left(1+y x_{1}\right)\left(1+y h_{n}\left(x_{2}, x_{3} \ldots, x_{n+1}, y\right)\right) \\
& =1+y\left(x_{1}+\left(1+x_{1} y\right) h_{n}\left(x_{2}, x_{3} \ldots, x_{n+1}, y\right)\right) .
\end{aligned}
$$

Now we conclude from the fact that our ring is an integral domain. 
Corollary 6 is the analogue of the formula (14) in [5, p. 148].

The first polynomials $h_{n}$ are

$$
\begin{aligned}
& h_{1}=x_{1}, \quad h_{2}=x_{1} y x_{2}+x_{1}+x_{2}, \\
& h_{3}=x_{1} y x_{2} y x_{3}+x_{1} y x_{2}+x_{1} y x_{3}+x_{2} y x_{3}+x_{1}+x_{2}+x_{3} .
\end{aligned}
$$

Thus

$$
h_{1}\left(x_{1}\right)=H_{1}\left(x_{1}\right), \quad h_{2}\left(x_{1}, x_{2}, y\right)=H_{2}\left(x_{1}, y, x_{2}\right),
$$

where $H_{n}$ denotes the continuant introduced in Section 2, but

$$
h_{3}\left(x_{1}, x_{2}, x_{3}, y\right) \neq H_{3}\left(x_{1}, y, x_{2}, y, x_{3}\right) .
$$

The product decomposition analogous to (2) and (8) for the polynomials $h_{n}$ is the decomposition

$$
\left(\begin{array}{cc}
x_{1} & 1 \\
1 & -y
\end{array}\right)\left(\begin{array}{ll}
y & 1 \\
1 & 0
\end{array}\right)\left(\begin{array}{cc}
x_{2} & 1 \\
1 & -y
\end{array}\right) \cdots\left(\begin{array}{cc}
y & 1 \\
1 & 0
\end{array}\right)\left(\begin{array}{cc}
x_{n} & 1 \\
1 & -y
\end{array}\right)=\left(\begin{array}{cc}
h_{n} & 1 \\
1 & -y
\end{array}\right) .
$$

The proof is by induction on $n$. The case $n=1$ is trivial. For the inductive step, it sufficies to check that

$$
\left(\begin{array}{cc}
h_{n} & 1 \\
1 & -y
\end{array}\right)\left(\begin{array}{ll}
y & 1 \\
1 & 0
\end{array}\right)\left(\begin{array}{cc}
x_{n+1} & 1 \\
1 & -y
\end{array}\right)=\left(\begin{array}{cc}
h_{n+1} & 1 \\
1 & -y
\end{array}\right)
$$

and this trivially follows from Proposition 5(b).

As usual, from the associativity of the product of matrices, we get the following formula, true for all $1 \leqslant l \leqslant n$ and relating $h_{n}, h_{l}$ and $h_{n-l}$ :

$$
h_{n}=h_{l}\left(y h_{n-l}\left(x_{l+1}, \ldots, x_{n}, y\right)+1\right)+h_{n-l}\left(x_{l+1}, \ldots, x_{n}, y\right),
$$

where $h_{n}=h_{n}\left(x_{1}, \ldots, x_{n}, y\right)$ and $h_{l}=h_{l}\left(x_{1}, \ldots, x_{l}, y\right)$.

The properties of these polynomials $h_{n}$ are very similar to the properties of the continuants studied in the previous sections. For instance, the analogues of the first formulae in $[5$, p. $148,(16)$ and (17)] are the equalities

$$
h_{n}\left(x_{1}, \ldots, x_{i-1}, 0, x_{i+1}, \ldots, x_{n}, y\right)=h_{n-1}\left(x_{1}, \ldots, x_{i-1}, \widehat{x}_{i}, x_{i+1}, \ldots, x_{n}, y\right)
$$

for every $i=1,2, \ldots, n$. To prove this, set $x_{i}=0$ in the defining formula (13). The second formula in $[5$, p. $148,(16)]$ is:

Lemma 7. $h_{n}\left(1, x_{2}, \ldots, x_{n}, y\right)=h_{n-1}\left(1+x_{2}+y x_{2}, x_{3}, \ldots, x_{n}, y\right)$.

Proof. From Corollary 6, we have that

$$
\begin{aligned}
h_{n-1}(1+ & \left.x_{2}+y x_{2}, x_{3}, \ldots, x_{n}, y\right) \\
& =1+x_{2}+y x_{2}+\left(1+\left(1+x_{2}+y x_{2}\right) y\right) h_{n-2}\left(x_{3}, \ldots, x_{n}, y\right) \\
& =1+(1+y) x_{2}+\left(1+y+x_{2} y+y x_{2} y\right) h_{n-2}\left(x_{3}, \ldots, x_{n}, y\right) \\
& =1+(1+y) x_{2}+(1+y)\left(1+x_{2} y\right) h_{n-2}\left(x_{3}, \ldots, x_{n}, y\right) \\
& =1+(1+y)\left(x_{2}+\left(1+x_{2} y\right) h_{n-2}\left(x_{3}, \ldots, x_{n}, y\right)\right) \\
& =1+(1+y) h_{n-1}\left(x_{2}, \ldots, x_{n}, y\right)=h_{n}\left(1, x_{2}, \ldots, x_{n}, y\right)
\end{aligned}
$$

by Corollary 6 again. 
Similarly, changing the sign of all the indeterminates $x_{1}, \ldots, x_{n}, y$ in (13), we see that

$$
h_{n}\left(-x_{1}, \ldots,-x_{n},-y\right)=-h_{n}\left(x_{1}, \ldots, x_{n}, y\right),
$$

which is the analogue of $[5$, p. $148,(19)]$.

All the polynomials $h_{n}$ are sums of monomials with all the coefficients equal to 1 . From Proposition 5(b), it is easily seen that each $h_{n}$ is a sum of monomials of all possible odd degrees $\leqslant 2 n-1$. Also, the number of monomials on the right hand side of (13) is $2^{n}$, so that $h_{n}$ is a sum of $2^{n}-1$ monomials, that is, $h_{n}(1,1, \ldots, 1 ; 1)=2^{n}-1$.

The results in this Section show that the polynomials $h_{n}$ have a behavior that is very similar to the behavior of the polynomials $H_{n}$ defined in Section 2 . It is very natural to ask what are the analogues of the polynomials $G_{n}$. The answer is that they are the noncommutative polynomials $q_{n}$ defined by

$$
q_{0}=1 \quad \text { and } \quad q_{n}=\left(1+x_{1} y\right)\left(1+x_{2} y\right) \ldots\left(1+x_{n} y\right) .
$$

We have that

$$
\left(\begin{array}{cc}
x_{1} & 1 \\
1 & -y
\end{array}\right)\left(\begin{array}{ll}
y & 1 \\
1 & 0
\end{array}\right)\left(\begin{array}{cc}
x_{2} & 1 \\
1 & -y
\end{array}\right) \cdots\left(\begin{array}{cc}
x_{n} & 1 \\
1 & -y
\end{array}\right)\left(\begin{array}{cc}
y & 1 \\
1 & 0
\end{array}\right)=\left(\begin{array}{cc}
q_{n} & h_{n} \\
0 & 1
\end{array}\right) .
$$

Each $q_{n}$ is a sum of $2^{n}$ distinct monomials, and $q_{n}(1,1, \ldots, 1 ; 1)=2^{n}$.

Similar results hold for the polynomials $q_{n}^{\prime}$ defined by $q_{0}^{\prime}=1$ and

$$
q_{n}^{\prime}\left(x_{1}, \ldots, x_{n}, y\right)=\left(1+y x_{1}\right)\left(1+y x_{2}\right) \ldots\left(1+y x_{n}\right) .
$$

Then $q_{n}^{\prime}=y h_{n}+1$ (Proposition 5(a)). Trivially,

$$
q_{n}^{\prime}\left(x_{1}, \ldots, x_{n}, y\right)=\left(1+y x_{1}\right) q_{n-1}^{\prime}\left(x_{2}, \ldots, x_{n}, y\right) .
$$

Moreover,

$$
\begin{gathered}
\left(\begin{array}{ll}
y & 1 \\
1 & 0
\end{array}\right)\left(\begin{array}{cc}
x_{1} & 1 \\
1 & -y
\end{array}\right)\left(\begin{array}{ll}
y & 1 \\
1 & 0
\end{array}\right)\left(\begin{array}{cc}
x_{2} & 1 \\
1 & -y
\end{array}\right) \cdots \\
\cdots\left(\begin{array}{ll}
y & 1 \\
1 & 0
\end{array}\right)\left(\begin{array}{cc}
x_{n} & 1 \\
1 & -y
\end{array}\right)=\left(\begin{array}{ll}
q_{n}^{\prime} & 0 \\
h_{n} & 1
\end{array}\right) .
\end{gathered}
$$

We collect some formulae for $q_{n}$ and $q_{n}^{\prime}$. We leave the easy proofs to the reader.

Lemma 8. (a) $q_{n}\left(x_{1}, \ldots, x_{n}, y\right)=h_{n}\left(x_{1}, \ldots, x_{n}, y\right) y+1$

(a') $q_{n}^{\prime}\left(x_{1}, \ldots, x_{n}, y\right)=y h_{n}\left(x_{1}, \ldots, x_{n}, y\right)+1$.

(b) $h_{n+1}\left(x_{1}, \ldots, x_{n+1}, y\right)=q_{n}\left(x_{1}, \ldots, x_{n}, y\right) x_{n+1}+h_{n}\left(x_{1}, \ldots, x_{n}, y\right)$.

(b') $h_{n+1}=x_{1} q_{n}^{\prime}\left(x_{2}, \ldots, x_{n}, y\right)+h_{n}\left(x_{2}, \ldots, x_{n}, y\right)$. 
(c) $q_{n}\left(x_{1}, \ldots, x_{i-1}, 0, x_{i+1}, \ldots, x_{n}, y\right)=q_{n-1}\left(x_{1}, \ldots, x_{i-1}, \widehat{x}_{i}, \ldots, x_{n-1}, y\right)$.

(c') $q_{n}^{\prime}\left(x_{1}, \ldots, x_{i-1}, 0, x_{i+1}, \ldots, x_{n}, y\right)=q_{n-1}^{\prime}\left(x_{1}, \ldots, x_{i-1}, \widehat{x}_{i}, x_{i+1}, \ldots, x_{n}, y\right)$.

(d) $q_{n}\left(-x_{1}, \ldots,-x_{n},-y\right)=q_{n}\left(x_{1}, \ldots, x_{n}, y\right)$.

(d') $q_{n}^{\prime}\left(-x_{1}, \ldots,-x_{n},-y\right)=q_{n}^{\prime}\left(x_{1}, \ldots, x_{n}, y\right)$.

(e) For $1 \leqslant l \leqslant n, q_{n}\left(x_{1}, \ldots, x_{n}, y\right)=q_{l}\left(x_{1}, \ldots, x_{l}, y\right) q_{n-l}\left(x_{l+1}, \ldots, x_{n}, y\right)$.

(e') For $1 \leqslant l \leqslant n, q_{n}^{\prime}\left(x_{1}, \ldots, x_{n}, y\right)=q_{l}^{\prime}\left(x_{1}, \ldots, x_{l}, y\right) q_{n-l}^{\prime}\left(x_{l+1}, \ldots, x_{n}, y\right)$.

(f) For $1 \leqslant l \leqslant n$,

$$
h_{n}\left(x_{1}, \ldots, x_{n}, y\right)=q_{l}\left(x_{1}, \ldots, x_{l}, y\right) h_{n-l}\left(x_{l+1}\left(x_{l+1}, \ldots, x_{n}, y\right)+h_{l}\left(x_{1}, \ldots, x_{l}, y\right)\right.
$$

(f') For $1 \leqslant l \leqslant n$,

$$
h_{n}\left(x_{1}, \ldots, x_{n}, y\right)=h_{l}\left(x_{1}, \ldots, x_{l}, y\right) q_{n-l}^{\prime}\left(x_{l+1}, \ldots, x_{n}, y\right)+h_{n-l}\left(x_{l+1}, \ldots, x_{n}, y\right) .
$$

\section{The graph algebra for the polynomials $h_{n}$}

The polynomials $h_{n}$ are also elements of a graph algebra with relations $k \Delta_{n} / I$. The quiver $\Delta_{n}$ is the following:

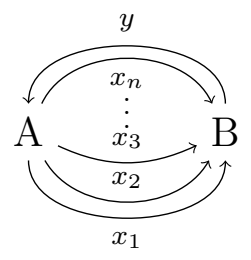

Thus the directed graph $\Delta_{n}$ has $n$ arrows $x_{1}, \ldots, x_{n}$ from $A$ to $B$ and one arrow $y$ from $B$ to $A$, so that $\Delta_{n}$ has $n+1$ arrows. Let $k$ be a field, consider the quiver algebra $k \Delta_{n}$ and the ideal $I$ of $k \Delta_{n}$ generated by all paths $x_{i} y x_{j}$ with $i \geqslant j$. The quotient algebra $k \Delta_{n} / I$ is a finite dimensional $k$-algebra, because the longest possible path not in $I$ is the path $y x_{1} y x_{2} y \ldots x_{n} y$ of length $2 n+1$. In particular, $R:=k_{n} / I$ is an artinian ring with $R / J(R) \cong k \times k$. The algebra $R=R_{0} \oplus R_{1}$ is 2-graded, where $R_{0}$ corresponds to the paths of even length and $R_{1}$ to the paths of odd length. In particular, the images of the polynomials $h_{n}$ in $R$ are all in $R_{1}$. The elements $x_{i} y$ are nilpotent of index 2 , hence they belong to $J(R)$, so $\sum_{i=1}^{n} x_{i} y$ is nilpotent, and $1-\sum_{i=1}^{n} x_{i} y$ is invertible.

Theorem 9. In the ring $R=k \Delta_{n} / I$, we have that:
(a) $h_{n}=\left(1-\sum_{i=1}^{n} x_{i} y\right)^{-1}\left(\sum_{i=1}^{n} x_{i}\right)$,
(b) $q_{n}=\left(1-\sum_{i=1}^{n} x_{i} y\right)^{-1}$,
(c) $h_{n}=\left(\sum_{i=1}^{n} x_{i}\right)\left(1-\sum_{i=1}^{n} y x_{i}\right)^{-1}$,
(d) $q_{n}^{\prime}=\left(1-\sum_{i=1}^{n} y x_{i}\right)^{-1}$, and
(e) $h_{n}\left(\sum_{i=1}^{n} y x_{i}\right)=\left(\sum_{i=1}^{n} x_{i} y\right) h_{n}$, for every $n \geqslant 1$. 
Proof. (a) It suffices to show that

$$
\left(1-\sum_{i=1}^{n} x_{i} y\right) h_{n}=\left(\sum_{i=1}^{n} x_{i}\right) .
$$

We do it by induction on $n \geqslant 1$. The case $n=1$ is easy, because

$$
\left(1-x_{1} y\right) h_{1}=\left(1-x_{1} y\right) x_{1}=x_{1} .
$$

Suppose our formula true for $n$. Then

$$
\begin{aligned}
& \left(1-\sum_{i=1}^{n+1} x_{i} y\right) h_{n+1} \\
& =\left(1-\sum_{i=1}^{n} x_{i} y-x_{n+1} y\right)\left(x_{n+1}+h_{n}\left(1+y x_{n+1}\right)\right) \\
& =\left(1-\sum_{i=1}^{n} x_{i} y\right) x_{n+1}+\left(1-\sum_{i=1}^{n} x_{i} y\right) h_{n}\left(1+y x_{n+1}\right)-x_{n+1} y h_{n}\left(1+y x_{n+1}\right) \\
& =x_{n+1}-\sum_{i=1}^{n} x_{i} y x_{n+1}+\left(\sum_{i=1}^{n} x_{i}\right)\left(1+y x_{n+1}\right) \\
& =\sum_{i=1}^{n+1} x_{i},
\end{aligned}
$$

which concludes the proof of (a).

(b) We will prove that $q_{n}\left(1-\sum_{i=1}^{n} x_{i} y\right)=1$ in $R$ by induction on $n \geqslant 1$. The case $n=1$ is trivial. Suppose the formula true for $n-1$. Then

$$
\begin{aligned}
& q_{n}\left(1-\sum_{i=1}^{n} x_{i} y\right)=q_{n-1}\left(1+x_{n} y\right)\left(1-\sum_{i=1}^{n} x_{i} y\right) \\
& \quad=q_{n-1}\left(1+x_{n} y-\sum_{i=1}^{n} x_{i} y\right)=q_{n-1}\left(1-\sum_{i=1}^{n-1} x_{i} y\right)=1
\end{aligned}
$$

by the inductive hypothesis.

(e) is easy and left to the reader.

(d) Like for (b), it suffices to show by induction on $n$ that

$$
q_{n}^{\prime}\left(1-\sum_{i=1}^{n} y x_{i}\right)=1
$$

(c) From (e) and (a), we have that

$$
h_{n}\left(1-\sum_{i=1}^{n} y x_{i}\right)=\left(1-\sum_{i=1}^{n} x_{i} y\right) h_{n}=\sum_{i=1}^{n} x_{i} .
$$

Thus (c) also holds. 
Again, in our graph $\Delta_{n}$ with two vertices $A$ and $B$, the paths leap alternatively from $A$ to $B$.

Proposition 10. The matrices $\left(\begin{array}{cc}x_{i} & 1 \\ 1 & -y\end{array}\right)(i=1,2, \ldots, n)$ and $\left(\begin{array}{ll}y & 1 \\ 1 & 0\end{array}\right)$ are invertible in the ring $R=k \Delta_{n} / I$ (that is, they are invertible elements of the ring $M_{2}(R)$ ). Their inverses are

$$
\left(\begin{array}{cc}
y-y x_{i} y & 1-y x_{i} \\
1-x_{i} y & -x_{i}
\end{array}\right) \quad \text { and } \quad P(0) P(-y) P(0)
$$

respectively.

\section{The generalized Fibonacci polynomials $f_{n}$}

We now generalize the continuants $p_{n}\left(t_{1}, t_{2}, \ldots, t_{n}\right)$ of Section 2 from the case of one sequence of indeterminates $t_{n}$ to the case of two sequences of indeterminates. The new polynomials we obtain appear in the study of generalized continued fractions, hence we call them generalized Fibonacci polynomials.

Our new polynomials $f_{n}$ are again polynomials with coefficients in $\mathbb{Z}$ and in the noncommutative indeterminates $x_{1}, x_{2}, x_{3}, \ldots$ and $y_{1}, y_{2}, y_{3}, \ldots$ They are defined by the recursion formulae:

$$
\begin{aligned}
& f_{-1}=0, \quad f_{0}=1, \\
& f_{n}\left(x_{1}, \ldots, x_{n}, y_{1}, \ldots, y_{n}\right)=f_{n-1}\left(x_{1}, \ldots, x_{n-1}, y_{1}, \ldots, y_{n-1}\right) x_{n} \\
& \quad+f_{n-2}\left(x_{1}, \ldots, x_{n-2}, y_{1}, \ldots, y_{n-2}\right) y_{n} .
\end{aligned}
$$

Thus, when we specialize all the indeterminates $y_{i}$ to 1 , these polynomials turn out to be the continuants $p_{n}$ of Section 2 in one countable set of indeterminates, that is, $p_{n}\left(t_{1}, \ldots, t_{n}\right)=f_{n}\left(t_{1}, \ldots, t_{n}, 1,1, \ldots, 1\right)$. Also,

$$
f_{n}(x, \ldots, x, 1,1, \ldots, 1)=F_{n}(x),
$$

the commutative Fibonacci polynomials, which have received much attention [3, Introduction]. Moreover, $f_{n}(x, \ldots, x, y, \ldots, y)$ turns out to be the noncommutative analogue of the generalized Fibonacci polynomial $\{n\}_{x, y}$ studied in [3].

The first of these polynomials $f_{n}$ are

$$
\begin{aligned}
& f_{0}=1, \quad f_{1}=x_{1}, \quad f_{2}=x_{1} x_{2}+y_{2}, \\
& f_{3}=x_{1} x_{2} x_{3}+x_{1} y_{3}+y_{2} x_{3}, \\
& f_{4}=x_{1} x_{2} x_{3} x_{4}+x_{1} x_{2} y_{4}+x_{1} y_{3} x_{4}+y_{2} x_{3} x_{4}+y_{2} y_{4}, \\
& \begin{aligned}
f_{5}= & x_{1} x_{2} x_{3} x_{4} x_{5}+x_{1} x_{2} x_{3} y_{5}+x_{1} x_{2} y_{4} x_{5}+x_{1} y_{3} x_{4} x_{5} \\
& \quad+x_{1} y_{3} y_{5}+y_{2} x_{3} x_{4} x_{5}+y_{2} x_{3} y_{5}+y_{2} y_{4} x_{5}, \ldots
\end{aligned}
\end{aligned}
$$

The number of monomials in each $f_{n}$ is the $(n+1)$-th Fibonacci number $F_{n+1}$.

These polynomials can be built using a leapfrog construction similar to that in Section 2 for continuants polynomials. For $f_{n}$, start writing the product $x_{1} \ldots x_{n}$ and add all the monomials obtained by replacing all the possible disjoint consecutive pairs $x_{i} x_{i+1}$ by $y_{i+1}$. 
Notice that the indeterminate $y_{1}$ does not appear in any polynomial

$$
f_{n}\left(x_{1}, \ldots, x_{n}, y_{1}, \ldots, y_{n}\right),
$$

that is, every $f_{n}\left(x_{1}, \ldots, x_{n}, y_{1}, \ldots, y_{n}\right)$ has degree 0 in the indeterminate $y_{1}$. Let us prove by induction on $n$ that

$$
f_{n}\left(x_{1}, \ldots, x_{n}, y_{1}, x_{1} x_{2}, x_{2} x_{3}, x_{3} x_{4}, \ldots, x_{n-1} x_{n}\right)=F_{n+1} x_{1} x_{2} \ldots x_{n} .
$$

For $n=-1$, we have that $f_{-1}=0=F_{0}$. For $n=0$, we have that $f_{0}=1=F_{1}$. Assume the result true for the indices smaller than $n$. Then

$$
\begin{aligned}
& f_{n}\left(x_{1}, \ldots, x_{n}, y_{1}, x_{1} x_{2}, x_{2} x_{3}, x_{3} x_{4}, \ldots, x_{n-1} x_{n}\right) \\
& =f_{n-1}\left(x_{1}, \ldots, x_{n-1}, y_{1}, x_{1} x_{2}, x_{2} x_{3}, x_{3} x_{4}, \ldots, x_{n-2} x_{n-1}\right) x_{n} \\
& \quad \quad \quad+f_{n-2}\left(x_{1}, \ldots, x_{n-2}, y_{1}, x_{1} x_{2}, x_{2} x_{3}, x_{3} x_{4}, \ldots, x_{n-3} x_{n-2}\right) x_{n-1} x_{n} \\
& \quad=F_{n} x_{1} x_{2} \ldots x_{n-1} \cdot x_{n}+F_{n-1} x_{1} x_{2} \ldots x_{n-2} \cdot x_{n-1} x_{n} \\
& \quad=F_{n+1} x_{1} x_{2} \ldots x_{n} .
\end{aligned}
$$

Let us mention another interesting specialization: if one puts $y_{i}=x_{i}$, for any $i=$ $1, \ldots, n$ we obtain new polynomials in the variables $x_{1}, \ldots, x_{n}$ :

$$
d_{n}\left(x_{1}, \ldots, x_{n}\right)=f_{n}\left(x_{1}, \ldots, x_{n}, x_{1}, \ldots, x_{n}\right) .
$$

In particular, we have $d_{0}=1, d_{1}\left(x_{1}\right)=x_{1}, d_{2}\left(x_{1}, x_{2}\right)=x_{1} x_{2}+x_{2}, d_{3}\left(x_{1}, x_{2}, x_{3}\right)=$ $x_{1} x_{2} x_{3}+x_{1} x_{3}+x_{2} x_{3}$. Specializing further, we get the sequence of natural numbers $D_{n}=d_{n}(0,1,2, \ldots, n-1)$ that gives the number of derangements in the symmetric group $S_{n}$. We will see that the polynomials $f_{n}$ admit a matrix presentation (cf. (20)). This easily leads to a presentation of the elements of the sequence $D_{n}$ (cf. also [8]).

The polynomials $f_{n}$ are also homogeneous polynomials of degree $n$ if we give all the indeterminates $x_{i}$ degree 1 and all the indeterminates $y_{i}$ degree 2. Thus, if we view $f_{n}\left(x_{1}, \ldots, x_{n}, y_{1}, \ldots, y_{n}\right)$ as an element of $k\left\langle x_{1}, \ldots, x_{n}, y_{1}, \ldots, y_{n}\right\rangle$, where $k$ is any commutative ring and $k\left\langle x_{1}, \ldots, x_{n}, y_{1}, \ldots, y_{n}\right\rangle$ is the free $k$-algebra in the noncommutative indeterminates $x_{1}, \ldots, x_{n}, y_{1}, \ldots, y_{n}$, then

$$
f_{n}\left(\lambda x_{1}, \ldots, \lambda x_{n}, \lambda^{2} y_{1}, \ldots, \lambda^{2} y_{n}\right)=\lambda^{n} f_{n}\left(x_{1}, \ldots, x_{n}, y_{1}, \ldots, y_{n}\right)
$$

for every $\lambda \in k$.

Finally, it is not difficult to see that $f_{n}(x, \ldots, x, y, \ldots, y)$ is the sum of all monic monomials of degree $n$ in the free algebra $\mathbb{Z}\langle x, y\rangle$ in the two indeterminates $x, y$ when the indeterminate $x$ is given degree 1 and $y$ is given degree 2. Two other formulae, that essentially appear in [3], are

$$
f_{n}(2,2, \ldots, 2,-1,-1, \ldots,-1)=n
$$

and, more generally,

$$
f_{n}(x+1, x+1, \ldots, x+1,-x,-x, \ldots,-x)=1+x+x^{2}+\cdots+x^{n-1} .
$$


These new polynomials $f_{n}$ are also entries of $2 \times 2$-matrices, as in the identity (2). Now we have that

$$
\begin{aligned}
& \left(\begin{array}{ll}
x_{1} & 1 \\
y_{1} & 0
\end{array}\right) \cdots\left(\begin{array}{ll}
x_{n} & 1 \\
y_{n} & 0
\end{array}\right) \\
& =\left(\begin{array}{cc}
f_{n}\left(x_{1}, \ldots, x_{n}, y_{1}, \ldots, y_{n}\right) & f_{n-1}\left(x_{1}, \ldots, x_{n-1}, y_{1}, \ldots, y_{n-1}\right) \\
y_{1} f_{n-1}\left(x_{2}, \ldots, x_{n}, y_{2}, \ldots, y_{n}\right) & y_{1} f_{n-2}\left(x_{2}, \ldots, x_{n-1}, y_{2}, \ldots, y_{n-1}\right)
\end{array}\right) .
\end{aligned}
$$

This is clear for $n=1$. The general case can be proved by induction, since on writing $f_{i}=f_{i}\left(x_{1}, \ldots, x_{i}, y_{1}, \ldots, y_{i}\right), f_{i}^{\prime}=f_{i}\left(x_{2}, \ldots, x_{i+1}, y_{2}, \ldots, y_{i+1}\right)$, we have that

$$
\left(\begin{array}{cc}
f_{n-1} & f_{n-2} \\
y_{1} f_{n-2}^{\prime} & y_{1} f_{n-3}^{\prime}
\end{array}\right)\left(\begin{array}{ll}
x_{n} & 1 \\
y_{n} & 0
\end{array}\right)=\left(\begin{array}{cc}
f_{n} & f_{n-1} \\
y_{1} f_{n-1}^{\prime} & y_{1} f_{n-2}^{\prime}
\end{array}\right)
$$

Regrouping the first $k$ matrices, for $1 \leqslant k \leqslant n$, in $(20)$, we get that the matrix on the right hand side of this equation is equal to the product

$$
\begin{aligned}
& \left(\begin{array}{cc}
f_{k}\left(x_{1}, \ldots, y_{k}\right) & f_{k-1}\left(x_{1}, \ldots, y_{k-1}\right) \\
y_{1} f_{k-1}\left(x_{2}, \ldots, y_{k}\right) & y_{1} f_{k-2}\left(x_{2}, \ldots, y_{k-1}\right)
\end{array}\right) \\
& \quad \cdot\left(\begin{array}{cc}
f_{n-k}\left(x_{k+1}, \ldots, y_{n}\right) & f_{n-k-1}\left(x_{k+1}, \ldots, y_{n-1}\right) \\
y_{k+1} f_{n-k-1}\left(x_{k+2}, \ldots, y_{n}\right) & y_{k+1} f_{n-k-2}\left(x_{k+2}, \ldots, y_{n-1}\right)
\end{array}\right)
\end{aligned}
$$

Comparing the $(1,1)$ entry of this product with the corresponding entry in $(20)$, we obtain:

$$
\begin{aligned}
f_{n}\left(x_{1}, \ldots, y_{n}\right)=f_{k}\left(x_{1},\right. & \left.\ldots, y_{k}\right) f_{n-k}\left(x_{k+1}, \ldots, y_{n}\right) \\
& +f_{k-1}\left(x_{1}, \ldots, y_{k-1}\right) y_{k+1} f_{n-k-1}\left(x_{k+2}, \ldots, y_{n}\right) .
\end{aligned}
$$

Let us now mention a few consequences of the equation (22). First we remark that for $k=1$, we have

$$
\begin{aligned}
& f_{n}\left(x_{1}, \ldots, x_{n}, y_{1}, \ldots, y_{n}\right) \\
& \quad=x_{1} f_{n-1}\left(x_{2}, \ldots, x_{n}, y_{2}, \ldots, y_{n}\right)+y_{2} f_{n-2}\left(x_{3}, \ldots, x_{n}, y_{3}, \ldots, y_{n}\right)
\end{aligned}
$$

The formula obtained in (22) can also be considered as a generalization of an analogous classical relation for the usual Fibonacci numbers $F_{n}$; that is, if one specializes $x_{1}=\cdots=x_{n}=y_{1}=\cdots=y_{n}=1$, we get that $F_{n}=F_{k} F_{n-k}+F_{k-1} F_{n-k-1}$.

Using the recursive relation in the definition of $f_{n}$ and the above equation (22), we easily obtain the following useful formula: for $1 \leqslant k<n$,

$$
\begin{aligned}
& f_{n}\left(x_{1}, x_{2}, \ldots, y_{n}\right) \\
& \quad=f_{k+1}\left(x_{1}, \ldots, x_{k}, f_{n-k}\left(x_{k+1}, \ldots, y_{n}\right), y_{1}, \ldots, y_{k}, f_{n-k-1}\left(x_{k+2}, \ldots, y_{n}\right)\right)
\end{aligned}
$$

In the free algebra $\mathbb{Z}<x_{1}, x_{2}, \ldots ; y_{1}, y_{2}, \cdots>$, we can define the standard partial derivations $\frac{\partial}{\partial x_{k}}$ and $\frac{\partial}{\partial y_{k}}$, for $k \geqslant 1$.

Using the equation (22), we then have the following: 


$$
\begin{array}{ll}
\frac{\partial f_{n}\left(x_{1}, \ldots, y_{n}\right)}{\partial x_{k}}=f_{k-1}\left(x_{1}, \ldots, y_{k-1}\right) f_{n-k}\left(x_{k+1}, \ldots, y_{n}\right), & \text { for } 1 \leqslant k \leqslant n . \\
\frac{\partial f_{n}\left(x_{1}, \ldots, y_{n}\right)}{\partial y_{k}}=f_{k-2}\left(x_{1}, \ldots, y_{k-2}\right) f_{n-k}\left(x_{k+1}, \ldots, y_{n}\right), & \text { for } 2 \leqslant k \leqslant n .
\end{array}
$$

As mentioned above, the polynomials $f_{n}$, specialized in $y_{1}=\cdots=y_{n}=1$, give the continuant polynomials $p_{n}$. In particular, it is easy to obtain formulas for the continuant polynomials analogous to the equations (22) and (25). On the other hand, we may as well specialize the polynomials $f_{n}$ in $x_{1}=\cdots=x_{n}=1$; we then get a family of polynomials $r_{n}\left(y_{1}, \ldots, y_{n}\right)$. The first values of these polynomials are $r_{1}=1, r_{2}=1+y_{2}, r_{3}=$ $1+y_{2}+y_{3}, r_{4}=1+y_{2}+y_{3}+y_{4}+y_{2} y_{4}, r_{5}=1+y_{2}+y_{3}+y_{4}+y_{5}+y_{2} y_{4}+y_{2} y_{5}+y_{3} y_{5}$. They satisfy the recurrence relation

$$
r_{n+1}\left(y_{1}, \ldots, y_{n+1}\right)=r_{n}\left(y_{1}, \ldots, y_{n}\right)+r_{n-1}\left(y_{1}, \ldots, y_{n-1}\right) y_{n+1} .
$$

In particular, if we specialize further, we obtain the following sequence of natural numbers $I_{n}=r_{n}(0,1, \ldots, n-1)$ which gives the number of involutions in the symmetric group $S_{n}$. As for earlier sequences, these can be presented using a specialization of the matrices in Equation (20) (cf. also [8]).

Using the polynomials $r_{n}$ and the equation (22), we easily obtain, for $1 \leqslant k \leqslant n$, that

$$
\begin{aligned}
f_{n}\left(1, \ldots, 1, x_{k+1}, \ldots, x_{n}, y_{1}, \ldots, y_{n}\right) & \\
=r_{k}( & \left.y_{1}, \ldots, y_{k}\right) x_{k+1} f_{n-k}\left(x_{k+1}, \ldots, y_{n}\right) \\
& +r_{k-1}\left(y_{1}, \ldots, y_{k-1}\right) y_{k+1} f_{n-k-1}\left(x_{k+2}, \ldots, y_{n}\right) .
\end{aligned}
$$

From (23), we get the formulae analogous to those given by P. M. Cohn for the continuant polynomials in [5, formulae (16), p. 148]:

$$
f_{n}\left(0, x_{2}, \ldots, x_{n}, y_{1}, \ldots, y_{n}\right)=y_{2} f_{n-2}\left(x_{3}, \ldots, x_{n}, y_{3}, \ldots, y_{n}\right)
$$

and

$$
f_{n}\left(1, x_{2}, \ldots, x_{n}, y_{1} \ldots y_{n}\right)=f_{n-1}\left(x_{2}+y_{2}, x_{3}, \ldots, x_{n}, y_{2}, y_{3}, \ldots, y_{n}\right)
$$

Conjugating all the matrices in the equation (20) by the invertible matrix $P(0)$, we find that

$$
\begin{aligned}
& \left(\begin{array}{cc}
0 & y_{1} \\
1 & x_{1}
\end{array}\right) \ldots\left(\begin{array}{cc}
0 & y_{n} \\
1 & x_{n}
\end{array}\right) \\
& =\left(\begin{array}{cc}
y_{1} f_{n-2}\left(x_{2}, \ldots, x_{n-1}, y_{2}, \ldots, y_{n-1}\right) & y_{1} f_{n-1}\left(x_{2}, \ldots, x_{n}, y_{2}, \ldots, y_{n}\right) \\
f_{n-1}\left(x_{1}, \ldots, x_{n-1}, y_{1}, \ldots, y_{n-1}\right) & f_{n}\left(x_{1}, \ldots, x_{n}, y_{1}, \ldots, y_{n}\right)
\end{array}\right) .
\end{aligned}
$$

We may also look at our noncommutative indeterminates $x_{1}, \ldots, x_{n}, y_{1}, \ldots, y_{n}$ in the polynomial $f_{n}\left(x_{1}, \ldots, x_{n}, y_{1}, \ldots, y_{n}\right)$ as arrows in a quiver $E_{n}$ with two vertices $A$ and $B$, where $x_{i}$ is an arrow from $A$ to $B$ for $i$ odd, $x_{i}$ is an arrow from $B$ to $A$ for $i$ even, $y_{i}$ is an arrow from $A$ to $A$ for $i$ odd, and $y_{i}$ is an arrow from $B$ to $B$ for $i$ even. The quiver $E_{n}$ is the following: 


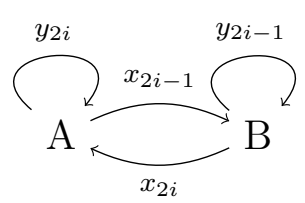

The quiver $E_{n}$ has $2 n$ arrows. The polynomials $f_{i}$ turn out to be linear combinations of paths with all the coefficients equal to one, and these paths in $f_{i}$ are from $A$ to $A$ (hence they are cycles) when $i$ is even, and are paths from $A$ to $B$ when $i$ is odd.

Notice that we have

$$
\left(\begin{array}{ll}
x_{2 i-1} & 1 \\
y_{2 i-1} & 0
\end{array}\right)\left(\begin{array}{c}
A \\
B
\end{array}\right)=\left(\begin{array}{c}
B \\
A
\end{array}\right) \text { and }\left(\begin{array}{ll}
x_{2 i} & 1 \\
y_{2 i} & 0
\end{array}\right)\left(\begin{array}{c}
B \\
A
\end{array}\right)=\left(\begin{array}{c}
A \\
B
\end{array}\right)
$$

so that again, in the product (20), the matrices alternatively leap from $A$ to $B$.

\section{Circular tilings and the polynomials $c_{n}$}

The classes of polynomials studied in this paper have a clear combinatorial interpretation. For instance:

(1) The monomials in the polynomial $h_{n}$ parametrize the nonempty subsets of a set of $n$ elements. It suffices to associate to the monomial $x_{i_{1}} y x_{i_{2}} y \ldots y x_{i_{t}}$ of $h_{n}$ the subset $\left\{i_{1}, i_{2}, \ldots, i_{t}\right\}$ of $\{1,2, \ldots, n\}$.

(2) The monomials in the polynomial

$$
f_{n}=f_{n}\left(x_{1}, \ldots, x_{n}, y_{1}, \ldots, y_{n}\right)
$$

studied in Section 6 parametrize the possible ways one can tile a strip of $1 \times n$ square cells with $1 \times 1$ squares and $1 \times 2$ dominos. Essentially, this is the standard interpretation of the Fibonacci numbers $F_{n}$ via linear tilings. A linear tiling of a row of squares $($ a $1 \times n$ strip of square cells) is a covering of the strip of squares with squares and dominos (which cover two squares). For instance, the polynomial $f_{3}=x_{1} x_{2} x_{3}+x_{1} y_{3}+y_{2} x_{3}$ parametrizes the set of the three linear tilings
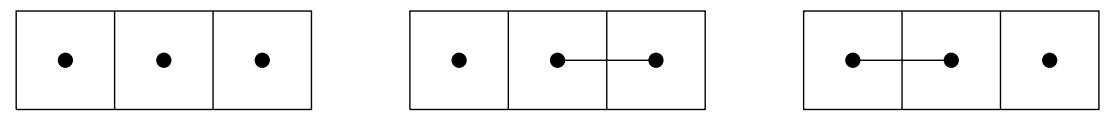

of a row of three squares. Here $x_{i}$ denotes the $i$-th square and $y_{i}$ denotes the domino that covers the $(i-1)$-th and the $i$-th square ( $y_{i}$ is the domino that "ends on the $i$-th square".) The Fibonacci number $F_{n}$ represents the number of tilings of a strip of length $n$ using length 1 squares and length 2 dominos.

It is also possible to consider circular tilings, where the deformed square are arranged in a circle [3]. For instance, the four possible circular tilings of a circle of three squares are 

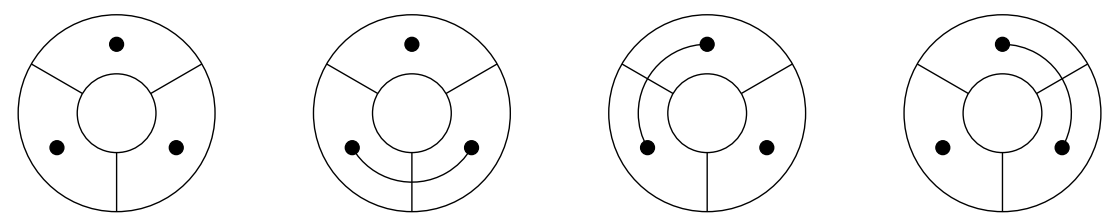

But for us it will be more convenient to represent the same four possible circular tilings of a circle of three squares as follows:
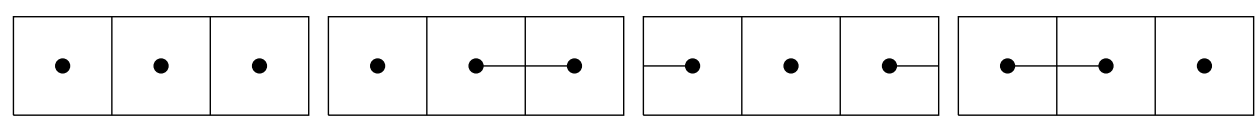

In this representation, we use an "out-of-phase domino" which spans the first and last cells of the tiling.

This suggests that there must also exist noncommutative polynomials $c_{n}$ which parametrize the set of circular tilings of a circle of $n$ squares. The idea is the following. Any circular tiling of a circle of $n$ squares $s_{1}, \ldots, s_{n}$ either does not contain the out-of-phase domino which spans $s_{n}$ and $s_{1}$ or contains the out-of-phase domino. The circular tilings of the circle that do not contain the out-of-phase domino are in one-to-one correspondence with the linear tilings of a row of squares $s_{1}, \ldots, s_{n}$, hence they are parametrized by the monomials of the polynomial $f_{n}\left(x_{1}, \ldots, x_{n}, y_{1}, \ldots, y_{n}\right)$. The circular tilings of the circle that do contain the out-of-phase domino are in one-to-one correspondence with the linear tilings of the row of squares $s_{2}, \ldots, s_{n-1}$, hence they are parametrized by the monomials of the polynomial

$$
f_{n-1}\left(x_{2}, \ldots, x_{n-1}, y_{2}, \ldots, y_{n-1}\right) .
$$

Now, as we have already said, the indeterminate $x_{i}$ denotes a length 1 square in the $i$-th position, and the indeterminate $y_{i}$ denotes a length 2 domino that ends in the $i$-th position. Thus we will denote the out-of-phase domino, which starts from the $n$-th position and ends in first one, by $y_{1}$. Hence we find that the circular tilings of a circle of $n$ squares $(n \geqslant 1)$ are parametrized by the noncommutative polynomials $c_{n}$ defined by

$$
\begin{aligned}
& c_{n}\left(x_{1}, \ldots, x_{n}, y_{1}, \ldots, y_{n}\right)= \\
& \quad=f_{n}\left(x_{1}, \ldots, x_{n}, y_{1}, \ldots, y_{n}\right)+y_{1} f_{n-2}\left(x_{2}, \ldots, x_{n-1}, y_{2}, \ldots, y_{n-1}\right) .
\end{aligned}
$$

Notice that:

(1) The indeterminate $y_{1}$ does not appear in the polynomials $f_{n}$, it appears in these new polynomials $c_{n}$ for the first time.

(2) In the polynomials $f_{n}(n \geqslant 1)$, all the monomials begin with $x_{1}$ or $y_{2}$ and end with $x_{n}$ or $y_{n}$. In the polynomials $c_{n}$, all the monomials begin with $x_{1}, y_{1}$ or $y_{2}$ and end with $x_{n-1}, y_{n-1}, x_{n}$ or $y_{n}$.

(3) The first polynomials $c_{n}$ are

$$
\begin{aligned}
& c_{1}\left(x_{1}, y_{1}\right)=x_{1}, \quad c_{2}\left(x_{1}, x_{2}, y_{1}, y_{2}\right)=x_{1} x_{2}+y_{1}+y_{2} \\
& c_{3}\left(x_{1}, x_{2}, x_{3}, y_{1}, y_{2}, y_{3}\right)=x_{1} x_{2} x_{3}+x_{1} y_{3}+y_{1} x_{2}+y_{2} x_{3} \\
& \begin{array}{r}
c_{4}\left(x_{1}, x_{2}, x_{3}, x_{4}, y_{1}, y_{2}, y_{3}, y_{4}\right)= \\
x_{1} x_{2} x_{3} x_{4}+x_{1} x_{2} y_{4}+x_{1} y_{3} x_{4} \\
+y_{1} x_{2} x_{3}+y_{1} y_{2}+y_{2} x_{3} x_{4}+y_{2} y_{4} .
\end{array}
\end{aligned}
$$


(4) Once again these polynomials can be obtained by a leapfrog construction as follows: to obtain $c_{n}$ you write $x_{1} \ldots x_{n}$ and replace every possible disjoint pairs $x_{i} x_{i+1}$ by $y_{i+1}$ (indexing module $n$ so that for a word terminating in $x_{n}$ and starting by $x-1$ the letter $x_{n}$ is erased and the letter $x_{1}$ is replaced by $\left.y_{1}\right)$.

(5) The polynomial $c_{2}\left(x_{1}, x_{2}, y_{1}, y_{2}\right)=x_{1} x_{2}+y_{1}+y_{2}$ parametrizes the three circular tilings
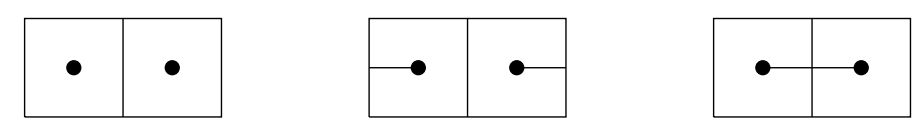

of a circle of two squares, which we considered to be distinct. With these conventions, we have that $c_{n}(1,1, \ldots, 1)=L_{n}$ for every $n \geqslant 1$, where $L_{n}$ indicates the $n$-th Lucas number, as desired. Here, the Lucas number $L_{n}$, defined by $L_{0}=2, L_{1}=1$ and $L_{n}=L_{n-1}+L_{n-2}$ when $n \geqslant 2$, represents for $n \geqslant 1$ the number of circular tilings of a strip of length $n$ using length 1 squares and length 2 dominos.

To relate the polynomials $c_{n}$ with a suitable product of matrices we need the trace. For any ring $R$, the abelian group $M_{n}(R)$ of all $n \times n$-matrices over $R$ can be viewed as an $R$ - $R$-bimodule ${ }_{R} M_{n}(R)_{R}$. The trace tr: ${ }_{R} M_{n}(R)_{R} \rightarrow{ }_{R} R_{R}$, defined by $\operatorname{tr}\left(a_{i j}\right)_{i, j}=\sum_{i=1}^{n} a_{i i}$, is an $R$-R-bimodule morphism with the further property that

$$
\operatorname{tr}\left(\left(a_{i j}\right)_{i, j}\left(b_{i j}\right)_{i, j}\right)=\sum_{i=1}^{n} \sum_{j=1}^{n} a_{i j} b_{j i} .
$$

From (20) and (29), we get that

\section{Theorem 11.}

$$
c_{n}\left(x_{1}, \ldots, x_{n}, y_{1}, \ldots, y_{n}\right)=\operatorname{tr}\left(\left(\begin{array}{ll}
x_{1} & 1 \\
y_{1} & 0
\end{array}\right) \cdots\left(\begin{array}{ll}
x_{n} & 1 \\
y_{n} & 0
\end{array}\right)\right)
$$

\section{The generalized Lucas polynomials $\ell_{n}$ and negative indices}

Now we can define the generalized Lucas polynomials by the recursion formulae:

$$
\begin{aligned}
& \ell_{0}=2, \quad \ell_{1}=x_{1}, \\
& \ell_{n}\left(x_{1}, \ldots, x_{n}, y_{1}, \ldots, y_{n}\right)=\ell_{n-1} \\
& \quad\left(x_{1}, \ldots, x_{n-1}, y_{1}, \ldots, y_{n-1}\right) x_{n} \\
& +\ell_{n-2}\left(x_{1}, \ldots, x_{n-2}, y_{1}, \ldots, y_{n-2}\right) y_{n},
\end{aligned}
$$

generalizing [4, p. 142].

The first of these polynomials are

$$
\begin{aligned}
\ell_{0} & =2, \quad \ell_{1}=x_{1}, \quad \ell_{2}=x_{1} x_{2}+2 y_{2}, \\
\ell_{3} & =x_{1} x_{2} x_{3}+x_{1} y_{3}+2 y_{2} x_{3}, \\
\ell_{4}= & x_{1} x_{2} x_{3} x_{4}+x_{1} x_{2} y_{4}+x_{1} y_{3} x_{4}+2 y_{2} x_{3} x_{4}+2 y_{2} y_{4}, \\
\ell_{5}= & x_{1} x_{2} x_{3} x_{4} x_{5}+x_{1} x_{2} x_{3} y_{5}+x_{1} x_{2} y_{4} x_{5}+x_{1} y_{3} x_{4} x_{5} \\
& \quad+x_{1} y_{3} y_{5}+2 y_{2} x_{3} x_{4} x_{5}+2 y_{2} x_{3} y_{5}+2 y_{2} y_{4} x_{5}, \ldots
\end{aligned}
$$


The number of monomials in each $\ell_{n}$ is the $(n+1)$-th Fibonacci number and, for $n \geqslant 1$, one has that $\ell_{n}(1,1, \ldots, 1)=L_{n}$, the $n$-th Lucas number. The polynomial

$$
\ell_{n}\left(x_{1}, \ldots, x_{n}, y_{1}, \ldots, y_{n}\right)
$$

belongs to $\mathbb{Z}\left\langle x_{1}, \ldots, x_{n}, y_{1}, \ldots, y_{n}\right\rangle$, though in this case also the indeterminate $y_{1}$ does not appear. But the polynomials $\ell_{n}$ are just a specialization of the polynomials $f_{n}$, as the following result shows.

\section{Theorem 12.}

$$
\ell_{n}\left(x_{1}, \ldots, x_{n}, y_{1}, \ldots, y_{n}\right)=f_{n}\left(x_{1}, x_{2}, x_{3} \ldots, x_{n}, y_{1}, 2 y_{2}, y_{3}, y_{4}, \ldots, y_{n}\right)
$$

for every $n \geqslant 1$.

Proof. Induction on $n$. The cases $n=1$ and $n=2$ are easily checked directly. For $n \geqslant 3$, assume that the theorem is true for $n-1$ and $n-2$. Then

$$
\begin{aligned}
& \ell_{n}\left(x_{1}, \ldots, x_{n}, y_{1}, \ldots, y_{n}\right) \\
& =\ell_{n-1}\left(x_{1}, \ldots, x_{n-1}, y_{1}, \ldots, y_{n-1}\right) x_{n}+\ell_{n-2}\left(x_{1}, \ldots, x_{n-2}, y_{1}, \ldots, y_{n-2}\right) y_{n} \\
& =f_{n-1}\left(x_{1}, \ldots, x_{n-1}, y_{1}, 2 y_{2}, \ldots, y_{n-1}\right) x_{n}+f_{n-2}\left(x_{1}, \ldots, x_{n-2}, y_{1}, 2 y_{2} \ldots, y_{n-2}\right) y_{n} \\
& =f_{n}\left(x_{1}, x_{2}, x_{3} \ldots, x_{n}, y_{1}, 2 y_{2}, y_{3}, y_{4}, \ldots, y_{n}\right) .
\end{aligned}
$$

Let us consider negative indices $n$. The sequence of Fibonacci numbers $F_{n}$ can be extended to any negative index $n$ using the recurrence formula $F_{n-2}=F_{n}-F_{n-1}$, and one finds that $F_{-n}=(-1)^{n+1} F_{n}$ for every $n \geqslant 0$. It is clear that our sequences of polynomials can be also extended to negative indices $n$.

Let us begin with the continuants $p_{n}\left(t_{1}, \ldots, t_{n}\right)$, for which we have that

$$
p_{n}(1,1, \ldots, 1)=F_{n+1} .
$$

The difference of 1 in the indices in this formula is due to Cohn's original choice of the initial conditions $p_{-1}=0, p_{0}=1$. Though the usual modern definition of Fibonacci numbers with $F_{0}=0, F_{1}=1$ is more appropriate, we prefer to continue using Cohn's original notation with $p_{-1}=0$ and $p_{0}=1$.

The recursion formula must now be re-written as $p_{n-2}=p_{n}-p_{n-1} t_{n}$. Substituting $n-2$ with $-m$, we get that $p_{-m}=p_{-(m-2)}-p_{-(m-1)} t_{-(m-2)}$. We thus find that $p_{0}=1, p_{-1}=$ $0, p_{-2}=1, p_{-3}=-t_{-1}, p_{-4}=t_{-1} t_{-2}+1$, and so on. The polynomial $p_{-n}$ for $n \geqslant 0$ turns out to be an element of the free algebra $\mathbb{Z}\left\langle t_{-1}, t_{-2}, \ldots, t_{-(n-2)}\right\rangle$ in the noncommutative indeterminates $t_{-1}, t_{-2}, \ldots, t_{-(n-2)}$. The general formula is given in the next proposition. Its proof is left to the reader.

Proposition 13.

$$
\begin{aligned}
p_{-n}\left(t_{-1}, t_{-2}, \ldots, t_{-(n-2)}\right) & =(-1)^{n} p_{n-2}\left(t_{-1}, t_{-2}, \ldots, t_{-(n-2)}\right) \\
& =p_{n-2}\left(-t_{-1},-t_{-2}, \ldots,-t_{-(n-2)}\right)
\end{aligned}
$$

for every integer $n \geqslant 0$. 
Notice that relation (2) now becomes

$$
\begin{aligned}
\left(\begin{array}{cc}
-t_{-1} & 1 \\
1 & 0
\end{array}\right) \cdots & \left(\begin{array}{cc}
-t_{-n} & 1 \\
1 & 0
\end{array}\right) \\
& =\left(\begin{array}{cc}
p_{-(n+2)}\left(t_{-1}, \ldots, t_{-n}\right) & p_{-(n-1)}\left(t_{-1}, \ldots, t_{-(n-1)}\right) \\
p_{-(n-1)}\left(t_{-2}, \ldots, t_{-n}\right) & p_{-n}\left(t_{-2}, \ldots, t_{-(n-1)}\right)
\end{array}\right) .
\end{aligned}
$$

For the generalized Fibonacci polynomials $f_{n}\left(x_{1}, x_{2}, \ldots, x_{n}, y_{1} \ldots y_{n}\right)$, we have that the recursion formula

$$
\begin{aligned}
f_{n}\left(x_{1}, \ldots, x_{n}, y_{1}, \ldots, y_{n}\right)=f_{n-1}( & \left.x_{1}, \ldots, x_{n-1}, y_{1}, \ldots, y_{n-1}\right) x_{n} \\
& +f_{n-2}\left(x_{1}, \ldots, x_{n-2}, y_{1}, \ldots, y_{n-2}\right) y_{n}
\end{aligned}
$$

now becomes

$$
\begin{aligned}
& f_{n-2}\left(x_{1}, \ldots, x_{n-2}, y_{1}, \ldots, y_{n-2}\right) \\
& =f_{n}\left(x_{1}, \ldots, x_{n}, y_{1}, \ldots, y_{n}\right) y_{n}^{-1}-f_{n-1}\left(x_{1}, \ldots, x_{n-1}, y_{1}, \ldots, y_{n-1}\right) x_{n} y_{n}^{-1} .
\end{aligned}
$$

Thus we get that, for any integer $n \geqslant 0$, the polynomial $f_{-n-2}$ must belong to the $\mathbb{Z}$-algebra $\mathbb{Z}\left\langle x_{-1}, x_{-2}, \ldots, x_{-n}, y_{0}^{ \pm 1}, y_{1}^{ \pm 1}, \ldots, y_{-(n-2)}^{ \pm 1}\right\rangle$, obtained from $\mathbb{Z}$ adjoining $2 n$ algebraically independent elements $x_{-1}, x_{-2}, \ldots, x_{-n}, y_{0}, y_{-1}, y_{-2}, \ldots, y_{-(n-1)}$ with $y_{0}, y_{-1}$, $\ldots, y_{-(n-1)}$ invertible. The proof of the following proposition, by induction, is left to the reader.

\section{Proposition 14.}

$$
\begin{aligned}
& f_{-n-2}\left(x_{-1}, x_{-2}, \ldots, x_{-n}, y_{0}, y_{1}, \ldots, y_{-(n-1)}\right) \\
& =f_{n}\left(-y_{0}^{-1} x_{-1},-y_{-1}^{-1} x_{-2}, \ldots,-y_{-(n-1)}^{-1} x_{-n}, 1, y_{0}^{-1}, y_{-1}^{-1}, \ldots, y_{-(n-2)}^{-1}\right) y_{-(n-2)}^{-1}
\end{aligned}
$$

for every integer $n \geqslant 0$.

In extending the generalized Lucas polynomials $\ell_{n}$ to negative indices $n$, we have, like for the $f_{n}$ 's in (32), that $\ell_{n-2}=\ell_{n} y_{n}^{-1}-\ell_{n-1} x_{n} y_{n}^{-1}$. It is easily seen that

\section{Proposition 15.}

$$
\begin{aligned}
& \ell_{-n}\left(x_{1}, x_{0}, x_{-1} \ldots, x_{-(n-2)}, y_{0}, y_{-1}, y_{-2}, \ldots, y_{-(n-2)}\right) \\
& =(-1)^{n} \ell_{n}\left(x_{1} y_{1}^{-1}, x_{0} y_{0}^{-1}, x_{-1} y_{-1}^{-1}, \ldots, x_{-(n-2)} y_{-(n-2)}^{-1} ; 1, y_{0}^{-1}, y_{-1}^{-1}, \ldots, y_{-(n-2)}^{-1}\right)
\end{aligned}
$$

for every integer $n \geqslant 0$.

\section{The general pattern: the polynomials $g_{n}$}

Now consider the following family of polynomials $g_{n}$, with $n \geqslant 0$. To define them, we need countably many noncommutative indeterminates $x_{i j}$, where $1 \leqslant i \leqslant j$. Set $g_{0}=1$ and

$$
g_{n}=\sum_{i=1}^{n} g_{i-1} x_{i n}, \quad \text { for } n \geqslant 1 .
$$


For instance, the first polynomials $g_{n}$ are

$$
\begin{gathered}
g_{1}=x_{11}, \quad g_{2}=x_{12}+x_{11} x_{22}, \quad g_{3}=x_{13}+x_{11} x_{23}+x_{12} x_{33}+x_{11} x_{22} x_{33}, \\
g_{4}=x_{14}+x_{11} x_{24}+x_{12} x_{34}+x_{11} x_{22} x_{34}+x_{13} x_{44}+x_{11} x_{23} x_{44} \\
+x_{12} x_{33} x_{44}+x_{11} x_{22} x_{33} x_{44} .
\end{gathered}
$$

For every $n \geqslant 1$, the polynomial $g_{n}$ turns out to be a polynomial with integer coefficients in the $n(n+1) / 2$ indeterminates $x_{i j}$ with $1 \leqslant i \leqslant j \leqslant n$, as is easily seen. The polynomial $g_{n}$ is a sum of monic monomials that parametrize all linear tilings of a strip of $n$ square cells, that is, all coverings of the strip of squares with rectangles of any length $1,2, \ldots, n$. The indeterminate $x_{i j}$ indicates the rectangle of length $j-i+1$ that starts covering the $i$-th square and ends covering the $j$-th square.

For instance, $g_{3}=x_{13}+x_{11} x_{23}+x_{12} x_{33}+x_{11} x_{22} x_{33}$ and, correspondingly, the tilings of a strip of three squares are
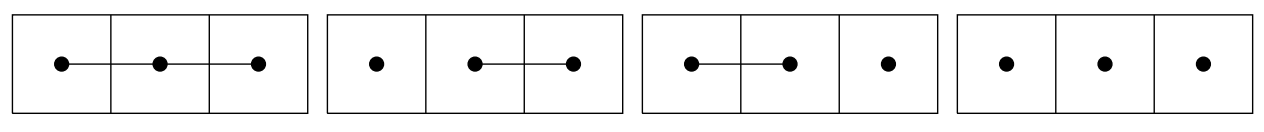

The first tiling consists of a unique rectangle of length 3. The second and the third of one rectangle of length 1 and one of length 2, in the two possible orders. The fourth tiling consists of three squares.

Remarks 16. (1) Let us show how we can recover the previous families of polynomials using the polynomials $g_{n}$. As we have seen, the family $f_{n}$ parametrizes tilings of a strip of length $n$ with tiles of length 1 (represented by the indeterminates $x_{i}$ in the definition of $f_{n}$ ) and of length 2 (represented by the indeterminates $y_{i}$ in the definition of $f_{n}$ ). It is clear from the equation (33) that $f_{n}$ can be obtained by equating, in this expression of $g_{n}$, all the indeterminates $x_{i j}$ to zero for $j>i+2$. In other words, the polynomial $f_{n}$ can be obtained from $g_{n}$ by specializing in the polynomial $g_{n}$ the indeterminates $x_{i j}$ to zero whenever $j>i+2$, the indeterminates $x_{i i}$ to $x_{i}$ and the indeterminates $x_{i, i+1}$ to $y_{i+1}$.

(2) Since the polynomials $p_{n}$ are obtained from $f_{n}$ by specializing the indeterminates $y_{i}$ (in the definition of $f_{n}$ ) to 1 and the indeterminates $x_{i}$ to $t_{i}$, we can also obtain the polynomials $p_{n}$ by specializing the indeterminates of the polynomial $g_{n}$. To be more precise this specialization is obtained by sending $x_{i j}$ to zero whenever $j>i+2, x_{i i}$ to $t_{i}$ and $x_{i, i+1}$ to 1 .

(3) The polynomial $g_{n}$ is the sum of $2^{n-1}$ monomials, which parametrize the subsets of a set of $n-1$ elements. Hence there is a clear immediate connection with the monomials of the polynomial $h_{n}$, which parametrize the nonempty subsets of a set of $n$ elements. To this end, it suffices to send every monomial $x_{i_{1} j_{1}} x_{i_{2} j_{2}} \ldots x_{i_{t-1} j_{t-1}} x_{i_{t} n}$ of degree $t$ in $g_{n}$ to the subset $\left\{j_{1}, j_{2}, \ldots, j_{t-1}\right\}$ of cardinality $t-1$ of the set $\{1,2, \ldots, n-1\}$. In order to get the polynomial $h_{n}$ from $g_{n+1}$, it suffices to specialize, in the polynomial $g_{n+1}$, the indeterminate $x_{i j}$ to $x_{j} y$ for every $i$ and $j$, then multiply by $\left(x_{n+1} y\right)^{-1}=y^{-1} x_{n+1}^{-1}$, subtract 1 , and finally multiply by $y^{-1}$ on the right. That is, after the specialization, we have that $h_{n}=\left(g_{n+1} y^{-1} x_{n+1}^{-1}-1\right) y^{-1}$. 
(4) The "hierarchy" of the polynomials we have studied in this paper follows therefore the following pattern. Each family of polynomials is a "specialization" of the families above it, as the remarks (1), (2), (3) above, Theorem 12 and (29) show.

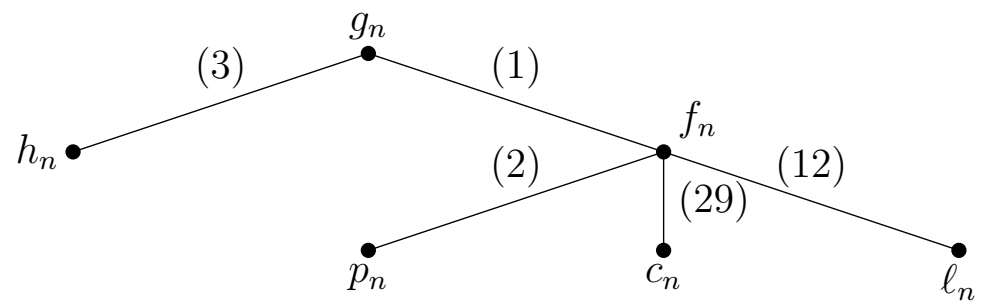

Notice that, in this diagram, $c_{n}$ is not really obtained via some specialization from $f_{n}$, because (29) is simply the definition of the $c_{n}$ 's, in terms of the polynomials $f_{n}$.

Equation (33) leads to

$$
\left(g_{1}, \ldots, g_{n}\right)=\left(g_{0}, \ldots, g_{n-1}\right)\left(\begin{array}{cccc}
x_{11} & x_{12} & \ldots & x_{1 n} \\
0 & x_{22} & \ldots & x_{2 n} \\
\vdots & \ddots & \ddots & \vdots \\
0 & \ldots & 0 & x_{n n}
\end{array}\right)
$$

Since, for $1 \leqslant l \leqslant n$, a tiling of a strip of length $n$ is obtained by a tile of length $l$ followed by a tiling of length $n-l$, the following formula, where we have specified explicitly the indeterminates ("the tiles") for each polynomial, is easy to get:

$$
g_{n}\left(x_{i j} ; 1 \leqslant i \leqslant j \leqslant n\right)=\sum_{l=1}^{n} x_{1 l} g_{n-l}\left(x_{l+i, l+j} ; 1 \leqslant i \leqslant j \leqslant n-l\right)
$$

The row $\left(g_{n}, \ldots, g_{1}\right)$ is also given by the first row of the following matrix product. This can be seen as a generalization of the equality (20).

$$
\left(\begin{array}{ccccc}
x_{11} & 1 & 0 & \ldots & 0 \\
* & 0 & 1 & 0 & \vdots \\
\vdots & \vdots & \ddots & & 0 \\
* & 0 & 0 & \ldots & 1 \\
* & 0 & 0 & \ldots & 0
\end{array}\right)\left(\begin{array}{ccccc}
x_{22} & 1 & 0 & \ldots & 0 \\
x_{12} & 0 & 1 & 0 & \vdots \\
* & 0 & \ddots & \ddots & 0 \\
\vdots & 0 & 0 & \ldots & 1 \\
* & 0 & 0 & \ldots & 0
\end{array}\right) \ldots\left(\begin{array}{ccccc}
x_{n n} & 1 & 0 & \ldots & 0 \\
x_{n-1, n} & 0 & 1 & 0 & \vdots \\
\vdots & \vdots & \ddots & & 0 \\
x_{2 n} & 0 & 0 & \ldots & 1 \\
x_{1 n} & 0 & 0 & \ldots & 0
\end{array}\right)
$$

\section{Permanents}

Let $R$ be a nonnecessarily commutative ring, $n \geqslant 1$ be an integer, $M_{n}(R)$ the $n \times n$-matrix ring, and $S_{n}$ be the symmetric group. Define a mapping perm: $M_{n}(R) \rightarrow R$ setting, for every matrix $A=\left(a_{i, j}\right)_{i, j} \in M_{n}(R)$,

$$
\operatorname{perm}(A):=\sum_{\sigma \in S_{n}} a_{1, \sigma(1)} \ldots a_{n, \sigma(n)} .
$$


If $A_{i, j}$ denotes the $(n-1) \times(n-1)$-matrix that results from $A$ removing the $i$-th row and the $j$-th column, then $\operatorname{perm}(A):=\sum_{j=1}^{n} a_{1, j} \operatorname{perm}\left(A_{1, j}\right)=\sum_{j=1}^{n} \operatorname{perm}\left(A_{n, j}\right) a_{n, j}$ (it is possible to easily expand our permanent along the first row or the last row only).

Theorem 17. For every $n \geqslant 1$,

$$
g_{n}\left(x_{i j}\right)=\operatorname{perm}\left(A_{n}\right)=\operatorname{perm}\left(A_{n}^{t}\right),
$$

where

$$
A_{n}=\left(\begin{array}{ccccc}
x_{11} & x_{12} & x_{13} & \ldots & x_{1 n} \\
1 & x_{22} & x_{23} & \ldots & x_{2 n} \\
0 & 1 & x_{33} & \ddots & x_{3 n} \\
\vdots & \ddots & \ddots & \ddots & \vdots \\
0 & \ldots & 0 & 1 & x_{n n}
\end{array}\right)
$$

Proof. Let us show that $g_{n}\left(x_{i j}\right)=\operatorname{perm}\left(A_{n}\right)$ by induction on $n$. The case $n=1$ is trivial.

Let us assume that the formula holds for $n$ and expand perm $\left(A_{n+1}\right)$ along the last row: $\operatorname{perm}\left(A_{n+1}\right)=\operatorname{perm}\left(B_{n}\right)+g_{n} x_{n+1, n+1}$ where $B_{n}$ is the $n \times n$ matrix given by $B_{n}=\left(\begin{array}{cc}A_{n-1} & C \\ U & x_{n, n+1}\end{array}\right)$, where $C$ is the column $\left(x_{1, n+1}, \ldots, x_{n-1, n+1}\right)^{t}$ and $U$ is the row $(0, \ldots, 0,1)$. In particular, the matrix $B_{n}$ is obtained from the matrix $A_{n}$ changing the last column. Notice that in the expression $g_{n}=\sum_{i=1}^{n} g_{i-1} x_{i n}$, the polynomials $g_{0}, \ldots, g_{n-1}$ do not depend on the indeterminates $x_{i n}$. Since the expression of $\operatorname{perm}\left(A_{n}\right)$ given by the inductive hypothesis is $\operatorname{perm}\left(A_{n}\right)=g_{n}$, we get that $\operatorname{perm}\left(B_{n}\right)=g_{0} x_{1, n+1}+g_{1} x_{2, n+1}+\cdots+$ $g_{n-1} x_{n, n+1}$. Thus we have $\operatorname{perm}\left(A_{n+1, n+1}\right)=\sum_{i=1}^{n+1} g_{i-1} x_{i, n+1}=g_{n+1}$, as desired. The fact that $\operatorname{perm}\left(A_{n}^{t}\right)=g_{n}$ as well is proved similarly, using the equality (34).

Let us remark that, contrary to the case of permanents defined over commutative rings, we do not have in general that $\operatorname{perm}(A)=\operatorname{perm}\left(A^{t}\right)$.

From Theorems 17, 12 and Remarks 16((1) and (2)), we immediately get that:

Corollary 18. The polynomials $f_{n}\left(x_{1}, \ldots, x_{n}, y_{1}, \ldots, y_{n}\right)$ and $p_{n}\left(t_{1}, \ldots, t_{n}\right)$ are the permanents of the $n \times n$ tridiagonal matrices

$$
\left(\begin{array}{ccccc}
x_{1} & y_{2} & 0 & \ldots & 0 \\
1 & x_{2} & y_{3} & \ldots & 0 \\
0 & 1 & x_{3} & \ddots & 0 \\
\vdots & \ddots & \ddots & \ddots & \vdots \\
0 & \ldots & 0 & 1 & x_{n}
\end{array}\right) \quad \text { and }\left(\begin{array}{ccccc}
t_{1} & 1 & 0 & \ldots & 0 \\
1 & t_{2} & 1 & \ldots & 0 \\
0 & 1 & t_{3} & \ddots & 0 \\
\vdots & \ddots & \ddots & \ddots & \vdots \\
0 & \ldots & 0 & 1 & t_{n}
\end{array}\right)
$$

and their transposes, respectively.

The analogue of formula (23) is the following: 


\section{Proposition 19.}

$$
\begin{array}{r}
g_{n}=x_{11} g_{n-1}\left(x_{i+1} j+1\right)+x_{12} g_{n-2}\left(x_{i+2} j+2\right)+x_{13} g_{n-3}\left(x_{i+3} j+3\right) \\
+\cdots+x_{n-1} g_{1}\left(x_{i+n-1} j+n-1\right)+x_{1 n} g_{0}
\end{array}
$$

Proof. It suffices to apply Theorem 17 expanding the permanent along the first row. The $t$-th term in this expansion is

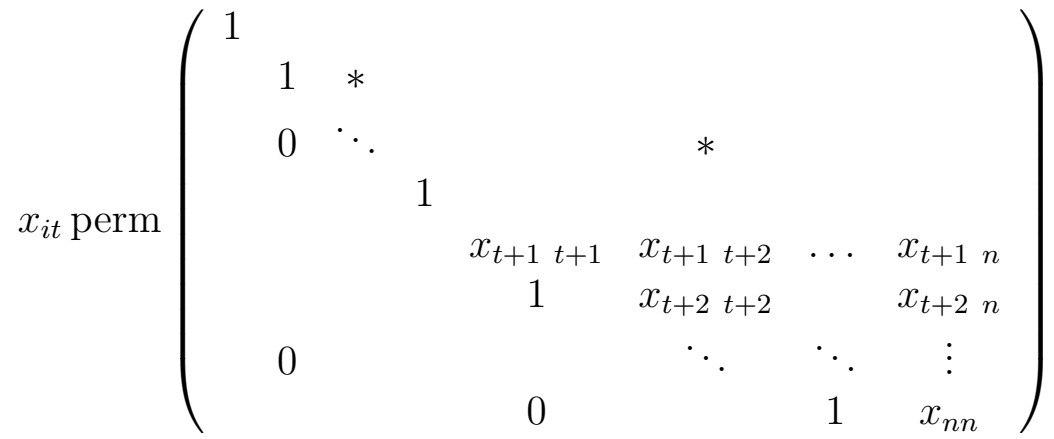

$$
\begin{aligned}
& =x_{i t} \operatorname{perm}\left(\begin{array}{cccc}
x_{t+1} t+1 & x_{t+1} t+2 & \ldots & x_{t+1 n} \\
1 & x_{t+2} t+2 & & x_{t+2 n} \\
& \ddots & \ddots & \vdots \\
0 & & 1 & x_{n n}
\end{array}\right)=x_{i t} g_{n-t}\left(x_{i+t j+t}\right) \text {. }
\end{aligned}
$$

\section{References}

[1] A. Alahmadi, S. K. Jain, A. Leroy, and T. Y. Lam. Euclidean pairs and quasi Euclidean rings. J. Algebra, 406:154-170, 2014.

[2] A. Alahmadi, and A. Facchini. Some remarks on categories of modules modulo morphisms with essential kernel or superfluous image. J. Korean Math. Soc., 50:557$578,2013$.

[3] T. Amdeberhan, X. Chen, V. H. Moll, and B. E. Sagan. Generalized Fibonacci polynomials and Fibonomial coefficients. Ann. Comb., 18:541-562, 2014.

[4] A. T. Benjamin, J. Quinn, and J. J. Quinn. Proofs that Really Count. Math. Assoc. Amer., 2003.

[5] P. M. Cohn. Free Ideal Rings and Localization in General Rings. Cambridge Univ. Press, Cambridge, 2006.

[6] P. M. Cohn. On the structure of the $G L_{2}$ of a ring. Inst. Hautes Études Sci. Publ. Math., 30:5-53, 1966.

[7] T. W. Cusick, and M. E. Flahive. The Markoff and Lagrange spectra. Math. Surveys and Monographs, no. 30, Amer. Math. Soc., Providence, RI, 1989.

[8] C. Reutenauer. On a matrix representation for polynomially recursive sequences. The Electronic Journal of Combinatorics, 19(3):\#P36, 2012.

[9] H. M. Wedderburn. Noncommutative domains of integrity. J. reine angew. Math., 167:129-141, 1932. 